\title{
Double-Framed Thin Elastomer Devices
}

\author{
Valeria Criscuolo, Nerio Andrés Montoya, Andrea Lo Presti, Luigi G. Occhipinti, Paolo Antonio Netti, \\ Raffaele Vecchione, and Christian Falconi*
}

Cite This: ACS Appl. Mater. Interfaces 2020, 12, 55255-55261

Read Online

ACCESS |

Шl Metrics \& More

Article Recommendations

Supporting Information

ABSTRACT: Elastomers and, in particular, polydimethylsiloxane (PDMS) are widely adopted as biocompatible mechanically compliant substrates for soft and flexible micro-nanosystems in medicine, biology, and engineering. However, several applications require such low thicknesses $(e . g$., $<100 \mu \mathrm{m})$ that make peeling-off critical because very thin elastomers become delicate and tend to exhibit strong adhesion with carriers. Moreover, microfabrication techniques such as photolithography use solvents which swell PDMS, introducing complexity and possible contamination, thus limiting industrial scalability and preventing many biomedical

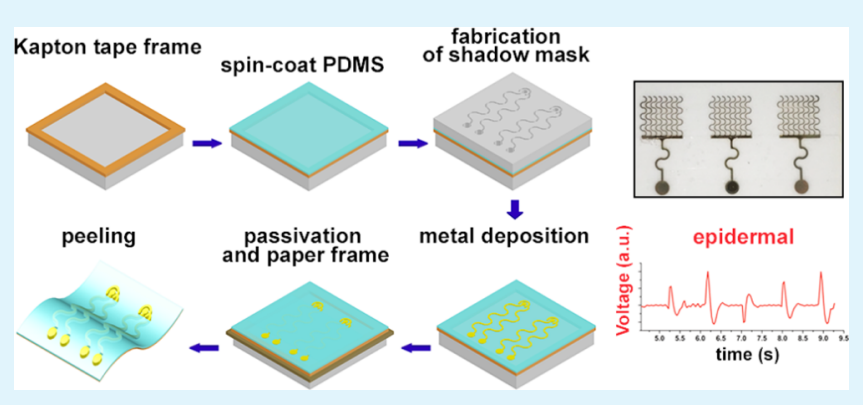
applications. Here, we combine low-adhesion and rectangular carrier substrates, adhesive Kapton frames, micromilling-defined shadow masks, and adhesive-neutralizing paper frames for enabling fast, easy, green, contaminant-free, and scalable manufacturing of thin elastomer devices, with both simplified peeling and handling. The accurate alignment between the frame and shadow masks can be further facilitated by micromilled marking lines on the back side of the low-adhesion carrier. As a proof of concept, we show epidermal sensors on a $50 \mu$ m-thick PDMS substrate for measuring strain, the skin bioimpedance and the heart rate. The proposed approach paves the way to a straightforward, green, and scalable fabrication of contaminant-free thin devices on elastomers for a wide variety of applications.

KEYWORDS: double-framed thin elastomer devices, contaminant-free epidermal devices, thin PDMS devices, epidermal electronics, peel-off, Kapton-paper frame

\section{INTRODUCTION}

Elastomers such as polydimethylsiloxane (PDMS) are widely used in medicine, biology, and engineering because of a unique combination of advantages, including excellent mechanical properties, ${ }^{1}$ easy processing, and biocompatibility. ${ }^{2}$ However, low thickness (e.g., below $100 \mu \mathrm{m})$ is a crucial requirement for several applications, including epidermal electronics, ${ }_{5}^{3,4}$ organs on chips, ${ }^{5}$ artificial skin models, ${ }^{6}$ microfluidic chips, ${ }^{7}$ molds for soft lithography, ${ }^{8}$ cell studies, ${ }^{9}$ tissue engineering, ${ }^{10}$ and membranes. ${ }^{1}$ Such very thin and soft films are extremely difficult to manufacture and to handle. In fact, both the strong adhesion between these thin films and their carriers and the unavoidable fragility associated with low thicknesses make the separation from the carrier by peel-off a critical step and limit both yield and reliability. Moreover, the typically high coefficient of thermal expansion (CTE) and the swelling characteristics of this class of elastomers introduce other restrictions. $^{12}$ For instance, volatile organic compounds, ${ }^{13}$ solvents, and small molecules can swell PDMS ${ }^{14,15}$ and penetrate the polymer structure for hundreds of micrometers $^{15,16}$ and then be released over time. ${ }^{13,15,17}$ Because of such limitations, thin PDMS devices often require timeconsuming and complex fabrication methods and may be not compatible with industrial fabrication and good manufacturing practices (GMP) needed for clinical applications. Some approaches may alleviate these issues. For instance, the removal of PDMS films can be facilitated by proper coating of the carrier ${ }^{18-20}$ or even by PDMS doping. ${ }^{21}$ As another example, an intermediate parylene layer ${ }^{22}$ before PDMS deposition enabled the safe removal of $150 \mu \mathrm{m}$-thick PDMS devices. Likewise, an intermediate polyimide (PI) layer, deposited on glass before PDMS, can be released by laser pulses through a transparent glass substrate. ${ }^{23}$ As an alternative, metals and, subsequently, PDMS can be, first, deposited on a substrate and, after a thermal procedure for strengthening the PDMS-metal adhesion, removed by conventional peeling. ${ }^{24}$ Nevertheless, these techniques can still be problematic because of, for instance, complex equipment, additional materials or steps, and unwanted materials changes or residuals. As an interesting approach, in principle, a frame $^{25-27}$ (or scaffold) can facilitate the peeling initiation and subsequent handling as well as reduce the risks of self-

Received: September 11, 2020

Accepted: November 9, 2020

Published: November 30, 2020 
a

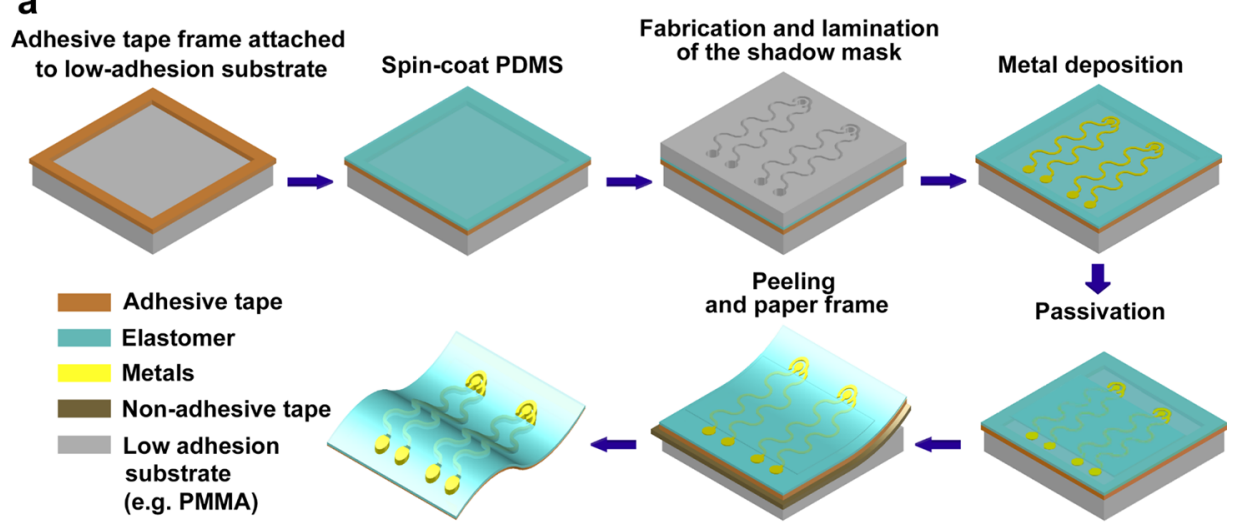

b

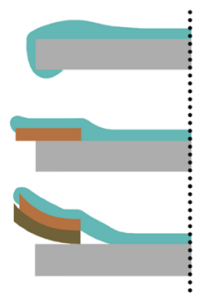

C

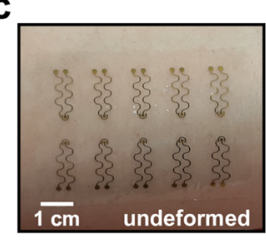

d

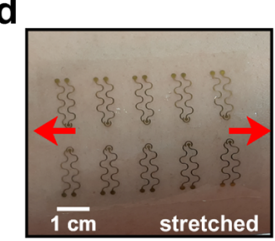

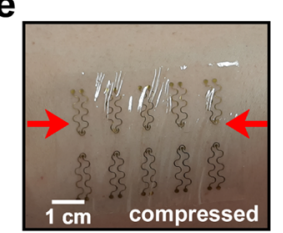

Figure 1. Double-framed contaminant-free thin elastomer devices. (a) Key fabrication steps. (b) Schematic cross section (not in scale) of the device border before removal from the substrate, emphasizing that the attachment of the elastomer to the back of the substrate (top) can be prevented by an auxiliary Kapton frame (center), whose adhesive can later be neutralized by a paper frame (bottom). (c-e) $50 \mu \mathrm{m}$-thick epidermal PDMS device on undeformed (c), stretched (d) and compressed (e) skin.

attachment, undesired contact with PDMS (i.e., contamination) and, if the frame is stiff enough, formation of wrinkles. However, despite these advantages, in case of PDMS devices, using frames, which must be placed on the periphery of the carrier before PDMS deposition and curing, introduces several issues. $^{25,26}$ First, the frame must be thin so that PDMS can cover the frame during spin coating. Such a thin frame should also be sufficiently stiff in order to avoid wrinkles after peeling but also not too stiff in order to not tear PDMS during peeling. Moreover, in case of a circular substrate, the frame should have an annular shape as sharp corners of the relatively stiff frame would easily tear the fragile PDMS during peeling. ${ }^{25,26}$ Clearly, manufacturing an annular, thin, and properly stiff frame, which should obviously withstand the PDMS curing temperature, can also be difficult. Additionally, for reliability, it may be necessary to reinforce PDMS in correspondence of the inner periphery of the frame. ${ }^{25,26}$ Finally, there are conflicting requirements for the frame-carrier adhesion, which should be very strong during fabrication (e.g., spin coating and metal evaporation) and very low during (so that low peel forces are sufficient) and after (so that the just detached frame does not self-attach) peeling.

Here, we combine rectangular and low-adhesion carriers, shadow masks, ${ }^{28,29}$ a first frame simply made of adhesive Kapton tape and an additional paper frame for solving these issues and enabling the contaminant-free, environmental friendly, fast and scalable fabrication of thin PDMS devices, as well as for simplifying peeling and subsequent handling. Besides, we take advantage of micromilling both to define the shadow masks and to facilitate the accurate alignment of the Kapton frame. As proofs of concept, we fabricated functional epidermal PDMS devices for measuring both the skin bioimpedance and the heart rate. The proposed strategy can obviously be generalized to other elastomers.

\section{EXPERIMENTAL SECTION}

Materials. PMMA foils of $0.5 \mathrm{~mm}$ and $1.1 \mathrm{~mm}$ were purchased from Good Fellow; $60 \mu$ m-thick and 12 mm-wide Kapton tape was purchased from TESA. PDMS (Sylgard 184) was purchased from Dow Corning. PMMA substrates and shadow masks were fabricated with a Minitech Mini Mill/GX (Minitech Machinery Corporation). PDMS thin films were spin coated with a LAURELL 650M SPIN COATER. Metal pellets were used as received from Kurt J. Lesker.

Fabrication. Kapton tape was attached to the PMMA carrier substrate to create the frame. A $50 \mu \mathrm{m}$ PDMS film (PDMS, Sylgard 184, Dow Corning, mixed at 10:1 (w/w) prepolymer:cross-linker) is then spin-coated onto the substrate $(1000 \mathrm{rpm}, 1 \mathrm{~min})$ and cured in oven at $65{ }^{\circ} \mathrm{C}$ for $2 \mathrm{~h}$. A bilayer of chromium and gold (10 and 200 $\mathrm{nm}$, respectively) is thermally evaporated on the PDMS film through a $500 \mu$ m-thick PMMA shadow mask which has been previously patterned with a micromilling machine. For passivation, we bonded a $25 \mu \mathrm{m}$ PDMS passivation film to the PDMS device. In detail, an auxiliary thick $3 \mathrm{~mm}$ PDMS slab is produced, and its surface is coated with a Fluorolink release layer after activation via oxygen plasma (50 $\mathrm{W}, 1 \mathrm{~min}$ ). The silanization solution of $1 \%$ Fluorolink is prepared as follows:

- $4,7 \mathrm{~mL}$ of isopropyl alcohol (94\%)

- $200 \mu \mathrm{L}$ of water (4\%)

- $50 \mu \mathrm{L}$ of acetic acid (1\%)

- $50 \mu \mathrm{L}$ of Fluorolink (1\%)

The $25 \mu \mathrm{m}$ passivation PDMS film is then spin-coated (2500 rpm, $1 \mathrm{~min}$ ) onto the Fluorolink-coated $3 \mathrm{~mm}$-thick auxiliary PDMS slab and cured at $65{ }^{\circ} \mathrm{C}$ for $3 \mathrm{~h}$. After activating both the top PDMS surface of the device and the free surface of the $25 \mu \mathrm{m}$ PDMS film with oxygen plasma, the double PDMS stack is flipped upside down, aligned to the device and brought in contact with the top PDMS surface of the device, resulting in PDMS-to-PDMS bonding. Finally, Fluorolink allows to easily detach the thick auxiliary PDMS slab from the $25 \mu \mathrm{m}$ PDMS film.

Characterization and Measurements. SEM images were acquired on a FESEM Ultraplus_ZEISS after coating the specimens with $5 \mathrm{~nm}$ of gold with a Cressington $208 \mathrm{HR}$ sputter coater. 

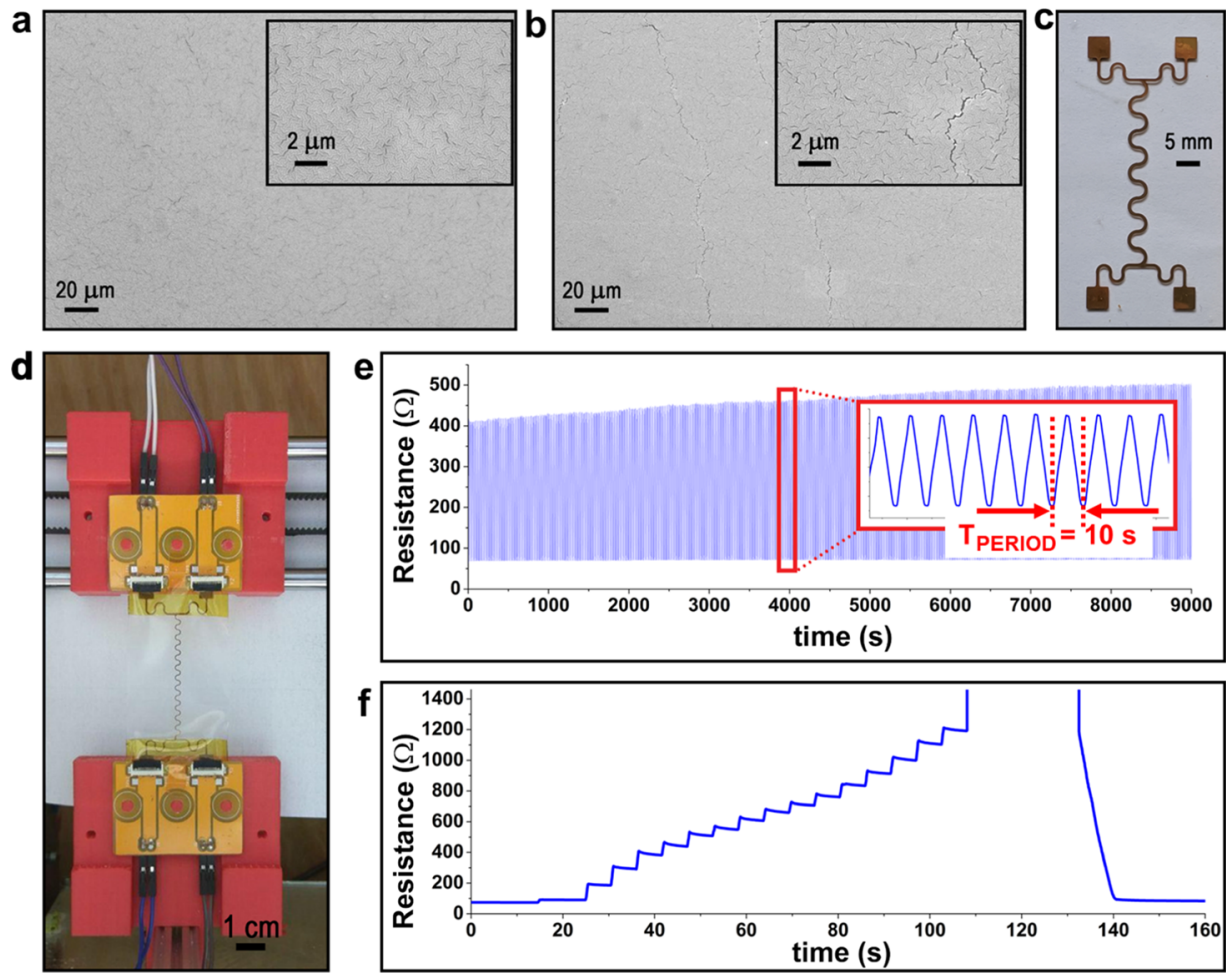

Figure 2. SEM characterization. (a,b) SEM image of a gold-coated $50 \mu \mathrm{m}$-thin PDMS device before (a) and after (b) peeling, showing that the gold film is not significantly damaged by peeling. (c) Photo of a $50 \mu \mathrm{m}$ PDMS device with a four-pad serpentine gold resistor. (d) Photo illustrating the system and the method for exerting a controlled strain to the device, with 3D printed holders (red) and PCBs (orange) for simplifying the four-wire connection to the external multimeter (see Figure S6). (e) Four-wire resistance measurements during strain cycles with $10 \%$ peak strain and $10 \mathrm{~s}$ period (see Figure S8 for details). (f) Four-wire resistance measurements when the strain is initially increased from zero, with $+1 \%$ strain steps, until the electrical continuity is lost (at strain $>16 \%$ ) and then reduced with $-1 \%$ steps, thus showing that the electrical continuity can be restored when the excessive strain is removed.

The bioimpedance measurements were performed by a commercial moisture meter (CMM) (Delfin Moisture Meter SC, Delfin Technologies Ltd.) and by our epidermal electrodes connected to an impedance analyzer AD5933 with $2 \mathrm{~V}$ (peak to peak) excitation and $[15 \mathrm{kHz}, 95 \mathrm{kHz}]$ frequency range, before and after applying the moisturizing lotion (Nivea Soft), respectively. Bioimpedances were measured after stabilization (e.g., due to sweating), and the measurement system was preliminarily calibrated by means of reference resistors and capacitors, as recommended in the AD5933 data sheet.

ECG measurements were performed using the AD8232 single-lead evaluation board with a three-electrode configuration. After acquiring the ECG signals with the electrodes placed on the chest and the central electrode as the reference, we applied a conventional digital filter for reducing noise and interferences. For validation, we compared ECG signals taken with our epidermal electrodes and with commercial pregelled $\mathrm{Ag} / \mathrm{AgCl}$ electrodes.

\section{RESULTS AND DISCUSSION}

Figure 1a schematically shows the key steps of the proposed fabrication method. In order to simplify peeling, we adopted carriers which have low adhesion with PDMS, such as poly(methyl methacrylate) (PMMA). In fact, the work of adhesion for PDMS/PMMA is as low as around $57 \mathrm{mN} /$ $\mathrm{m} .{ }^{30,31}$ Moreover, we employed rectangular carriers, so that, instead of manufacturing the frame, we simply attached a correspondent Kapton adhesive tape with a thickness and width equal to $60 \mu \mathrm{m}$ and $12 \mathrm{~mm}$, respectively, on each peripheral side of the PMMA carrier. After attaching the Kapton frame on the PMMA carrier, we spin-coated and cured the thin elastomer. The proposed strategy is general and applies to any elastomer and thickness. For validation, we considered a $50 \mu \mathrm{m}$ PDMS film which would be easily damaged by conventional methods. Afterward, we used 500 $\mu \mathrm{m}$ PMMA sheets, patterned with micromilling, as shadow masks ${ }^{28,29}$ for the selective deposition of metals on PDMS. As a key advantage, not only shadow masks can be quickly custom-made without expensive and time-consuming photolithographic steps but also there is no need for etching metals with aggressive solvents which can penetrate the bulk of PDMS and be subsequently released over very long times. ${ }^{14-17} \mathrm{~A}$ thin chromium adhesion layer and then gold were thermally evaporated on PDMS. Subsequently, the device can be passivated with different methods, including spray coating and PDMS-PDMS bonding. As schematically shown in Figure S1, we bonded a $25 \mu \mathrm{m}$ PDMS passivation film, previously attached to an auxiliary Fluorolink-coated $3 \mathrm{~mm}$-thick PDMS slab, and the final $50 \mu \mathrm{m}$ (Figure S2) PDMS device by oxygen plasma. After passivation, we initiated the peeling with a sidepeel-and-neutralize phase (Figure S3), which consists, for each side of the rectangular Kapton frame, in detaching Kapton from PMMA and then neutralizing the adhesive below the Kapton tape by attaching a correspondent auxiliary paper frame. Once the side-peel-and-neutralize procedure has been repeated for all the four sides, the PDMS film is double-framed by a Kapton-paper structure, which is entirely detached from the PMMA substrate, so that the device can be easily released from the PMMA carrier, independently on the strength of the Kapton tape adhesive. The proposed strategy allows to mechanically release functional thin PDMS devices without 
the key issues of conventional frames. ${ }^{25,26}$ First, by adopting rectangular carriers, instead of complex procedures for manufacturing proper annular scaffolds, frames can be simply placed by attaching conventional adhesive (e.g., Kapton) tapes at the periphery of the carrier before PDMS deposition and curing. Besides avoiding any frame manufacturing, there are adhesive tapes with different materials, properties, and thicknesses, thus allowing selection of the optimal frame for the specific application. For our tests, we used Kapton tapes which are available with several thicknesses (e.g., from 7.5 to $125 \mu \mathrm{m})$, can typically withstand PDMS curing temperatures and are stiff enough to avoid the risk of wrinkles but not so stiff that PDMS can be easily broken. In fact, we did not experience any issues and there was no need to reinforce PDMS in correspondence of the inner periphery of the frame. Rectangular (instead of circular) carriers and proper (e.g., Kapton) tapes easily solve all the key issues of mechanically released framed thin PDMS devices, ${ }^{25,26}$ except the conflicting requirements for the frame-carrier adhesion. In fact, the strong adhesive of the frame, required for firmly keeping the frame in position during fabrication (e.g., spin coating and metal evaporation), becomes problematic during and after peeling, as high peel forces are necessary for detaching the frame from the carrier and the adhesive can easily attach to PDMS or self-attach after detachment from the carrier. These difficulties are removed by the side-peel-and-neutralize procedure. In fact, after the adhesive of the Kapton tape has been covered, for each side of the frame, by paper, the PDMS film peeling only requires the force for detaching PDMS from the low-adhesion PMMA carrier and not the much stronger force required for detaching the adhesive frame from the carrier. After being attached to the Kapton adhesive, a properly selected paper can temporarily (until the double frame is simply cut away) give the device a certain degree of rigidity, thus greatly simplifying handling (Figure S3). Although chemical methods such as proper coating of the carrier ${ }^{18-20}$ or PDMS doping ${ }^{21}$ can reduce the PDMS-to-carrier adhesion, a much easier solution is to directly adopt carriers, which have relatively low adhesion with PDMS, such as parylene ${ }^{22}$ or PMMA. ${ }^{32,33}$ In our tests, we used PMMA carriers for significantly reducing the peel forces. The side-peel-andneutralize step is straightforward because of the rectangular shape of the carrier and, therefore, of the frames only if, as schematically shown in Figure 1b, the external border of the Kapton frame extends beyond the substrate ${ }^{25,26}$ so that PDMS may not strongly attach to the back of the carrier and peeling can be easily initiated without risks of PDMS damages. For an ideal alignment (e.g., errors below $0.5{ }^{\circ} \mathrm{C}$ ) of the Kapton tapes, because (Figure 1b) the external borders of Kapton must extend beyond the external border of the carrier, marking lines can optionally be micromilled on the back side of the PMMA carrier to guide the attachment of Kapton (Figure S4). Most remarkably, the proposed double-frame strategy minimizes pollution and does not require toxic chemicals. Figure S5 shows a typical Kapton-paper double frame we have used for our tests and devices. The final contaminant-free and robust PDMS devices have such a low adhesion with the PMMA carrier substrate that a skilled operator can manually release thin functional PDMS devices. As an example, Figure 1c shows a $50 \mu$ m-thick (Figure S2) stretchable and conformable epidermal PDMS device which, even when stretched or compressed, remains self-attached to the skin by van der Waals forces.
Figure 2 shows the SEM analysis of the gold traces of a device immediately after fabrication (Figure 2a) and after manual peeling (Figure 2b). Similar to previous reports, ${ }^{28,29}$ metal films directly deposited on elastomers are easily cracked even by the relatively small strains occurring during fabrication (e.g., due to the different coefficients of thermal expansion of elastomers and metals) or peeling. Nevertheless, such cracks, which can have lengths up to hundreds of micrometer and nanoscale widths, only result in increased resistances but do not affect the electrical continuity, which is generally preserved up to strains (e.g., above 10\%) which, in most cases, are sufficient for the implementation of flexible interconnects or devices. $^{28,29}$ For preliminary tests, we fabricated $50 \mu \mathrm{m}$ PDMS devices, each having a serpentine gold resistor with four pads for enabling high-accuracy four-wire resistance measurements (Figure 2c). In order to systematically characterize the metals deposited on PDMS (Figure 2d), after cutting away the double frame, we applied Kapton tape for both restricting the strain region as desired and for locally increasing the thickness of the devices in correspondence to the pads area, so that standard SMT (surface-mount technology) ZIF (zero insertion force) FPC (flexible printed circuit) connectors can establish good electrical connections between the pads of the device and conventional PCBs. A double-side adhesive tape is then sufficient to firmly fix the device to the PCBs (orange parts) which include the copper tracks for connecting to external instrumentation (e.g., multimeter) and also have holes for easily assembling to ad hoc designed 3D-printed holders (red block), which transmit the motion of a linear mechanical actuator (Figure S6). The negligible relative motion between the connectors and the pads prevents the connectors from scratching the delicate thin metal pads. After initial short-term tests with 50 strain cycles having peak strains equal to $2.5,5$, 7.5 , and $10 \%$ (Figure S7), as shown in Figure 2e,f, we performed four-wire resistance measurements during strain cycles with $10 \%$ peak strain and $10 \mathrm{~s}$ period (Figures $2 \mathrm{e}$ and S8). We also performed four-wire resistance measurements when the strain is initially increased from zero, with $+1 \%$ strain steps, until the electrical continuity is lost (at strain $>16 \%$ ) and then reduced with $-1 \%$ steps, thus showing that the electrical continuity can be restored when the excessive strain is removed (Figure 2f). The reversible open-circuit behavior (Figure 2f) and the gradual increase of the gauge factor (Figures S7, S8) are consistent with the expected straininduced formation and growth in the gold film of microcracks. These results confirm that the devices can be used as highperformance flexible interconnects and as ultrasensitive microcrack-based strain sensors. ${ }^{34}$ Moreover, these measurements show that the devices can remain functional not only after several high-strain (e.g., 10\%, Figures 2e, S7-S8) cycles but also after the transient application of strains, which can temporarily break the electrical continuity (Figure 2f). As an additional test, we gradually increased the strain until the device was broken at a $92.5 \%$ strain (according to the data sheet, the elongation at break for SYLGARD 184 is around $140 \%$, with possible variabilities ascribable to samples, fabrication procedures, and operating conditions). Figure S9 shows SEM images of metals after strain, thus confirming the formation of cracks in the metal thin film, consistent with resistance measurements (Figure 2e,f) and literature.

As proofs of concept, we also fabricated two different $50 \mu \mathrm{m}$ PDMS epidermal devices (Figure S10). In both cases, in order to minimize the risks of mechanical failure upon stretching, we 

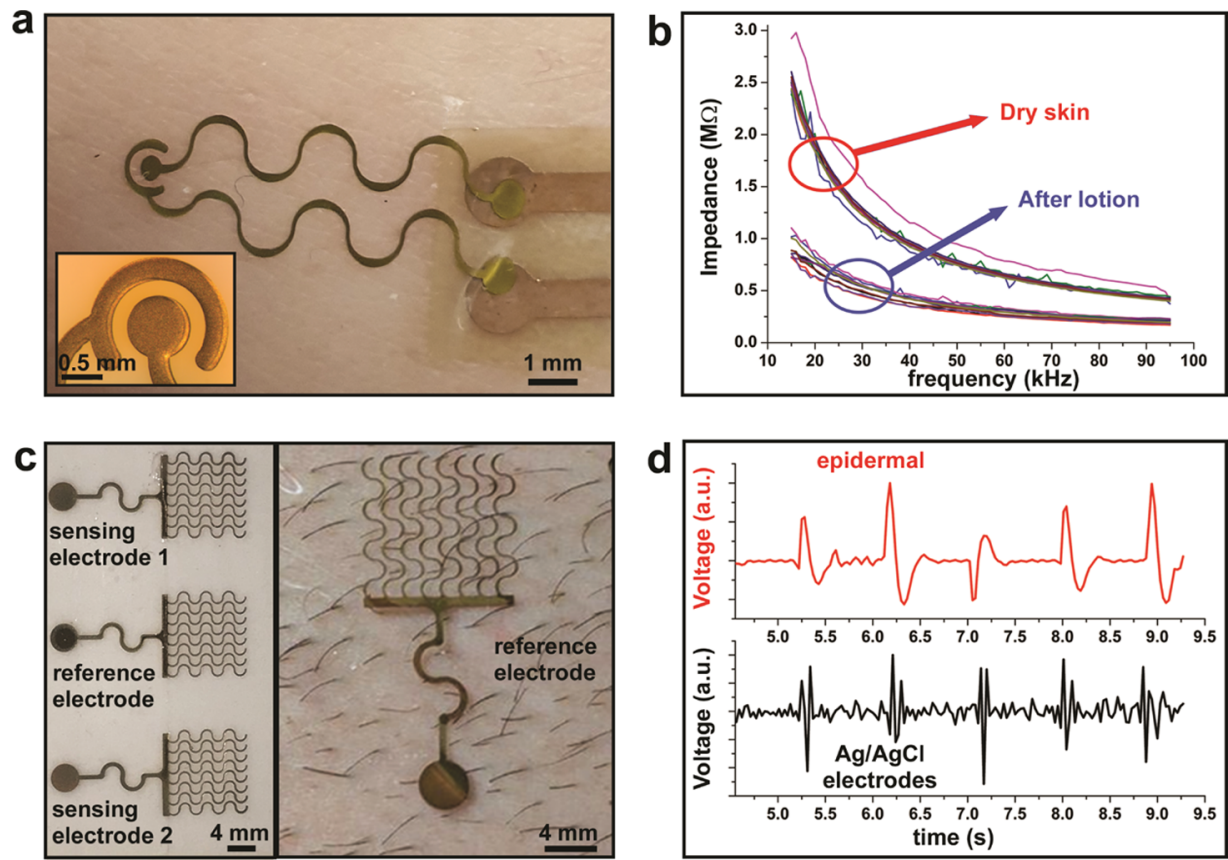

Figure 3. Epidermal devices. (a) Photo of the bioimpedance epidermal device on skin and (b) bioimpedance measurements taken on 10 electrodes. (c) Photo of the epidermal electro-cardiographic device (left) and of the reference electrode on skin (right) and (d) measured voltages taken with the epidermal electrodes and with conventional $\mathrm{Ag} / \mathrm{AgCl}$ electrodes.

took advantage of serpentine metal traces ${ }^{35}$ with a $1 / 3$ ribbonwidth-to-arc-radius ratio ${ }^{36}$ defined on the PMMA shadow masks by micromilling. As a first example, Figure 3a shows an epidermal bioimpedance sensor, which is one within an array of 10 sensors (Figure $1 \mathrm{c}-\mathrm{e}$ ) attached to the skin, with the sensor connected to the external electronics by conventional flexible PCB (printed circuit board) connectors. The inset shows one sensing element composed of a planar concentric pair of electrodes, with an inner disk electrode $(400 \mu \mathrm{m}$ radius), an outer arc electrode $(600 \mu \mathrm{m}$ inner radius, $900 \mu \mathrm{m}$ outer radius), and a $200 \mu \mathrm{m}$ distance between the two electrodes. This epidermal bioimpedance sensor can be used for evaluating the skin hydration, which is an important parameter in dermatology (e.g., eczema, acne, itching, and cracking of the stratum corneum) and can help to determine the effectiveness of therapies and cosmetic treatments. ${ }^{37}$ In fact, both the conductivity and the permittivity of the skin strongly depend on the skin hydration. ${ }^{37}$ The device was tested in vivo on the forearm of a healthy volunteer in two different hydration states, namely, skin without any treatment and skin moisturized by a commercial lotion (Nivea Soft). For comparison, we also evaluated the skin with a CMM and found 25 and 50 values (arbitrary units) before and after applying the moisturizing lotion, respectively. The measurements were performed by an impedance analyzer AD5933, with a $2 \mathrm{~V}_{\mathrm{pp}}$ excitation signal and a $[15 \mathrm{kHz}, 95 \mathrm{kHz}]$ frequency range, that is typical for similar measurements. ${ }^{38}$ Figure $3 \mathrm{~b}$ shows that all the 10 electrodes in the array worked properly (Table S1 shows the individual responses of the 10 electrodes). When the device is laminated onto the skin, before applying the lotion, we found an average (10 electrodes) impedance amplitude of $\sim 2.5 \mathrm{M} \Omega$ at $15 \mathrm{kHz}$ that decreased to $\sim 0.4 \mathrm{M} \Omega$ at $95 \mathrm{kHz}$. After moisturizing the skin, the average impedance amplitude significantly decreased to $\sim 900$ and $\sim 200 \mathrm{k} \Omega$ at 15 and $95 \mathrm{kHz}$, respectively. The bioimpedance changes are higher at the lower frequency $(15 \mathrm{kHz})$, as expected. $^{38}$ As a second example, we fabricated three epidermal electrodes (Figure 3c, left) for performing ECG measurements. The electrodes were placed on the chest, with the central electrode (Figure 3c, right) as the reference. During the measurements, the electrodes were connected to a conventional electronic interface (AD8232 single-lead, heart rate monitor front end with a three-electrode configuration). For validation, Figure $3 \mathrm{~d}$ shows both the ECG signals measured with our epidermal electrodes, after filtering, and the correspondent signals taken with commercial pregelled Ag/ $\mathrm{AgCl}$ electrodes, thus confirming that in both cases, the heart rate can be clearly detected.

\section{CONCLUSIONS}

The fabrication of contaminant-free elastomer devices with low thicknesses $($ e.g. $<100 \mu \mathrm{m})$ required by several applications is typically challenging, mainly because peeling off is very critical because of both the fragility of thin elastomers and their strong adhesion with carriers. Additionally, there may be other restrictions, which further complicate fabrication. For instance, many solvents used for photolithography swell PDMS and, after penetrating deep into the PDMS bulk, can later be slowly released over long times, which is generally not compliant with the often mandatory GMP. These problems generally result in complex processes and limit industrial scalability. The combination of low-adhesion and rectangular carrier substrates, adhesive Kapton frames, micromilled shadow masks, and adhesive-neutralizing paper frames can enable the fast, easy, green, contaminant-free, and scalable manufacturing of thin elastomer (e.g., PDMS) devices. These procedures facilitate peeling and subsequent handling, so that even manual peeling allows the release of functional devices. As examples, we have demonstrated the simple fabrication of $50 \mu$ m-thick contaminant-free PDMS devices with gold thin films as serpentine resistive strain sensors, bioimpedance, and heartrate epidermal sensors. These experiments already demonstrate 
that double-framed thin elastomer devices can be easily and effectively fabricated. However, the strategies reported here are much more general and can be applied to different tapes (materials, thicknesses, adhesives, etc.), metals, elastomers, and nonadhesive substrates, including possible modifications for tuning their properties. ${ }^{21,39}$ In our preliminary tests, we manually peeled $50 \mu \mathrm{m}$ PDMS devices from their PMMA carriers without any functional issues. However, clearly, any automatized method for releasing elastomer devices from their carriers with controlled velocities or forces or peeling angles can be applied to double-framed devices, which may be especially useful in case of much more fragile (e.g., significantly thinner) devices. The double-framed approach can greatly simplify the GMP-compliant fabrication of thin and contaminant-free soft and flexible devices for a wide variety of applications, including epidermal electronics, organs on chip, skin models, microfluidics, soft lithography, tissue engineering, and membranes.

\section{ASSOCIATED CONTENT}

\section{s) Supporting Information}

The Supporting Information is available free of charge at https://pubs.acs.org/doi/10.1021/acsami.0c16312.

Complete process for fabricating double-framed contaminant-free thin PDMS devices; SEM cross-section image of a $50 \mu \mathrm{m}$ PDMS device; side-by-side adhesive neutralization by auxiliary (non-adhesive) paper frame; optical image showing how marking lines micromilled on the carrier can reduce the Kapton tapes misalignment to very low angles; optical microscopic image of the Kapton-paper double frame and of the complete PDMSKapton-paper structure; system for strain characterization; strain characterization of thin gold resistors on PDMS; analysis of 4-wires resistance measurements taken during strain cycles with $10 \%$ peak strain; SEM images of metals after strain cycles; schematic diagrams (top view) of the process for electrodes fabrication and bioimpedance measurements taken with the 10 electrodes of a single epidermal device before (left column) and after (right column) treatment with a commercial moisturizing lotion (PDF)

\section{AUTHOR INFORMATION}

\section{Corresponding Author}

Christian Falconi - Department of Electronic Engineering, University of Rome Tor Vergata, Roma 00133, Italy; ○ orcid.org/0000-0002-5220-4588; Email: falconi@ eln.uniroma2.it

\section{Authors}

Valeria Criscuolo - Department of Electronic Engineering, University of Rome Tor Vergata, Roma 00133, Italy; Center for Advanced Biomaterial for Health Care, Istituto Italiano di Tecnologia, Naples 80125, Italy

Nerio Andrés Montoya - Department of Electronic Engineering, University of Rome Tor Vergata, Roma 00133, Italy; School of Physics, National University of Colombia, 3840, Colombia

Andrea Lo Presti - Department of Electronic Engineering, University of Rome Tor Vergata, Roma 00133, Italy
Luigi G. Occhipinti - Cambridge Graphene Centre, Department of Engineering, University of Cambridge, Cambridge CB3 OFA, United Kingdom

Paolo Antonio Netti - Center for Advanced Biomaterial for Health Care, Istituto Italiano di Tecnologia, Naples 80125, Italy

Raffaele Vecchione - Center for Advanced Biomaterial for Health Care, Istituto Italiano di Tecnologia, Naples 80125, Italy; $\odot$ orcid.org/0000-0002-8831-7891

Complete contact information is available at: https://pubs.acs.org/10.1021/acsami.0c16312

\section{Notes}

The authors declare no competing financial interest.

\section{ACKNOWLEDGMENTS}

This work has been supported by the Italian Ministry of Education, University and Research (MIUR-PRIN 2015 $20157 \mathrm{CCS} 5 \mathrm{X}-\mathrm{H}_{2} \mathrm{O}$ ). The experiments shown in Figure ${ }^{3} \mathrm{c}, \mathrm{d}$ have also been supported by a Regional Operative Program (POR CAMPANIA FESR 2014/2020-REMIAM project).

\section{REFERENCES}

(1) Rogers, J. A.; Someya, T.; Huang, Y. Materials and Mechanics for Stretchable Electronics. Science 2010, 327, 1603-1607.

(2) Bélanger, M.-C.; Marois, Y. Hemocompatibility, Biocompatibility, Inflammatory and in Vivo Studies of Primary Reference Materials Low-Density Polyethylene and Polydimethylsiloxane: A Review. J. Biomed. Mater. Res. 2001, 58, 467-477.

(3) Kim, D.-H.; Lu, N.; Ma, R.; Kim, Y.-S.; Kim, R.-H.; Wang, S.; Wu, J.; Won, S. M.; Tao, H.; Islam, A.; Yu, K. J.; Kim, T.-i.; Chowdhury, R.; Ying, M.; Xu, L.; Li, M.; Chung, H.-J.; Keum, H.; McCormick, M.; Liu, P.; Zhang, Y.-W.; Omenetto, F. G.; Huang, Y.; Coleman, T.; Rogers, J. A. Epidermal Electronics. Science 2011, 333, $838-843$.

(4) Xu, S.; Jayaraman, A.; Rogers, J. A. Skin Sensors Are the Future of Health Care. Nature 2019, 571, 319-321.

(5) Quirós-Solano, WF; Gaio, N.; Stassen, OMJA; Arik, YB; Silvestri, C.; Van Engeland, NCA; Van der Meer, A.; Passier, R.; Sahlgren, CM; Bouten, CVC; van den Berg, A.; Dekker, R.; Sarro, PM Microfabricated Tuneable and Transferable Porous PDMS Membranes for Organs-on-Chips. Sci. Rep. 2018, 8, 13524.

(6) Nachman, M.; Franklin, S. E. Artificial Skin Model Simulating Dry and Moist in Vivo Human Skin Friction and Deformation Behaviour. Tribol. Int. 2016, 97, 431-439.

(7) Thorsen, T.; Maerkl, S. J.; Quake, S. R. Microfluidic Large-Scale Integration.Pdf. Science 2002, 298, 580-584.

(8) Vozzi, G.; Flaim, C. J.; Bianchi, F.; Ahluwalia, A.; Bhatia, S. Microfabricated PLGA Scaffolds: A Comparative Study for Application to Tissue Engineering. Mater. Sci. Eng. C 2002, 20, 4347.

(9) Jiang, X.; Whitesides, G. M. Engineering Microtools in Polymers to Study Cell Biology. Eng. Life Sci. 2003, 3, 475-480.

(10) Khademhosseini, A.; Langer, R.; Borenstein, J.; Vacanti, J. P. Microscale Technologies for Tissue Engineering and Biology. Proc. Natl. Acad. Sci. U.S.A. 2006, 103, 2480-2487.

(11) Firpo, G.; Angeli, E.; Repetto, L.; Valbusa, U. Permeability Thickness Dependence of Polydimethylsiloxane (PDMS) Membranes. J. Membr. Sci. 2015, 481, 1-8.

(12) Tan, E.; Jing, Q.; Smith, M.; Kar-Narayan, S.; Occhipinti, L. Needs and Enabling Technologies for Stretchable Electronics Commercialization. MRS Adv. 2017, 2, 1721-1729.

(13) Tan, E. K. W.; Rughoobur, G.; Rubio-Lara, J.; Tiwale, N.; Xiao, Z.; Davidson, C. A. B.; Lowe, C. R.; Occhipinti, L. G. Nanofabrication of Conductive Metallic Structures on Elastomeric Materials. Sci. Rep. 2018, 8, 1-9. 
(14) Lee, J. N.; Park, C.; Whitesides, G. M. Solvent Compatibility of Poly(Dimethylsiloxane)-Based Microfluidic Devices. Anal. Chem. 2003, 75, 6544-6554.

(15) Adiraj Iyer, M.; Eddington, D. T. Storing and Releasing Rhodamine as a Model Hydrophobic Compound in Polydimethylsiloxane Microfluidic Devices. Lab Chip 2019, 19, 574-579.

(16) Toepke, M. W.; Beebe, D. J. PDMS Absorption of Small Molecules and Consequences in Microfluidic Applications. Lab Chip 2006, 6, 1484-1486.

(17) Mukhopadhyay, R. When PDMS Isn't the Best. Anal. Chem. 2008, 79, 3248-3253.

(18) Byun, I.; Ueno, R.; Kim, B. Micro-Heaters Embedded in PDMS Fabricated Using Dry Peel-off Process. Microelectron. Eng. 2014, 121, $1-4$.

(19) Byun, I.; Coleman, A. W.; Kim, B. SAM Meets MEMS: Reliable Fabrication of Stable Au-Patterns Embedded in PDMS Using Dry Peel-off Process. Microsyst. Technol. 2014, 20, 1783-1789.

(20) Vudayagiri, S.; Skov, A. L. Methods to Ease the Release of Thin Polydimethylsiloxane Films from Difficult Substrates. Polym. Adv. Technol. 2014, 25, 249-257.

(21) Vudayagiri, S.; Junker, M. D.; Skov, A. L. Factors Affecting the Surface and Release Properties of Thin Polydimethylsiloxane Films. Polym. J. 2013, 45, 871-878.

(22) Chou, N.; Jeong, J.; Kim, S. Crack-Free and Reliable Lithographical Patterning Methods on PDMS Substrate. J. Micromech. Microeng. 2013, 23, 125035.

(23) Joshi, S.; Savov, A.; Dekker, R. Substrate Transfer Technology for Stretchable Electronics. Procedia Eng. 2016, 168, 1555-1558.

(24) Koh, D.; Wang, A.; Schneider, P.; Bosinski, B.; Oh, K. W. Introduction of a Chemical-Free Metal PDMS Thermal Bonding for Fabrication of Flexible Electrode by Metal Transfer onto PDMS. Micromachines 2017, 8, 1-16.

(25) Krishnan, S. On the Manufacture of Very Thin Elastomeric Films by Spin-Coating; Massachusetts Institute of Technology, 2007.

(26) Krishnan, S.; Sarma, S. System and Method for Providing the Capability of Peeling Thin Polymer Films from a Substrate. U.S. Patent 0,109,199 A1, 2010.

(27) Lai, S.; Zucca, A.; Cosseddu, P.; Greco, F.; Mattoli, V.; Bonfiglio, A. Ultra-Conformable Organic Field-Effect Transistors and Circuits for Epidermal Electronic Applications. Org. Electron. 2017, $46,60-67$.

(28) Lacour, S. P.; Wagner, S.; Huang, Z.; Suo, Z. Stretchable Gold Conductors on Elastomeric Substrates. Appl. Phys. Lett. 2003, 82, 2404-2406.

(29) Lacour, S. P.; Jones, J.; Wagner, S.; Teng Li, T.; Zhigang Suo, Z. Stretchable Interconnects for Elastic Electronic Surfaces. Proc. IEEE 2005, 93, 1459-1467.

(30) Tian, Y.; Ina, M.; Cao, Z.; Sheiko, S. S.; Dobrynin, A. V. How to Measure Work of Adhesion and Surface Tension of Soft Polymeric Materials. Macromolecules 2018, 51, 4059-4067.

(31) Mangipudi, V. S.; Huang, E.; Tirrell, M.; Pocius, A. V. Measurement of Interfacial Adhesion between Glassy Polymers Using the JKR Method. Macromol. Symp. 1996, 102, 131-143.

(32) Kim, K.; Park, S. W.; Yang, S. S. The Optimization of PDMSPMMA Bonding Process Using Silane Primer. BioChip J. 2010, 4, $148-154$.

(33) Norouzi, A. R.; Nikfarjam, A.; Hajghassem, H. PDMS-PMMA Bonding Improvement Using $\mathrm{SiO} 2$ Intermediate Layer and Its Application in Fabricating Gas Micro Valves. Microsyst. Technol. 2018, 24, 2727-2736.

(34) Kang, D.; Pikhitsa, P. V.; Choi, Y. W.; Lee, C.; Shin, S. S.; Piao, L.; Park, B.; Suh, K. Y.; Kim, T. I.; Choi, M. Ultrasensitive Mechanical Crack-Based Sensor Inspired by the Spider Sensory System. Nature 2014, 516, 222.

(35) Pan, T.; Pharr, M.; Ma, Y.; Ning, R.; Yan, Z.; Xu, R.; Feng, X.; Huang, Y.; Rogers, J. A. Experimental and Theoretical Studies of Serpentine Interconnects on Ultrathin Elastomers for Stretchable Electronics. Adv. Funct. Mater. 2017, 27, 1702589.
(36) Widlund, T.; Yang, S.; Hsu, Y.-Y.; Lu, N. Stretchability and Compliance of Freestanding Serpentine-Shaped Ribbons. Int. J. Solids Struct. 2014, 51, 4026-4037.

(37) Huang, X.; Cheng, H.; Chen, K.; Zhang, Y.; Zhang, Y.; Liu, Y.; Zhu, C.; Ouyang, S.; Kong, G.; Yu, C.; Huang, Y.; Rogers, J. A. Epidermal Impedance Sensing Sheets for Precision Hydration Assessment and Spatial Mapping. IEEE Trans. Biomed. Eng. 2013, $60,2848-2857$.

(38) Huang, X.; Yeo, W. H.; Liu, Y.; Rogers, J. A. Epidermal Differential Impedance Sensor for Conformal Skin Hydration Monitoring. Biointerphases 2012, 7, 52.

(39) Deshpande, T. D.; Singh, Y. R. G.; Patil, S.; Joshi, Y. M.; Sharma, A. Adhesion Strength and Viscoelastic Properties of Polydimethylsiloxane (PDMS) Based Elastomeric Nanocomposites with Embedded Electrospun Nanofibers. Soft Matter 2019, 15, 57395747. 


\section{Supporting Information}

\section{Double-framed thin elastomer devices}

Valeria Criscuolo ${ }^{1,2}$, Nerio Andrés Montoya ${ }^{1,3}$, Andrea Lo Presti ${ }^{1}$, Luigi G. Occhipinti ${ }^{4}$,

Paolo Antonio Netti ${ }^{2}$, Raffaele Vecchione ${ }^{2}$, Christian Falconi $^{1 *}$

${ }^{1}$ Department of Electronic Engineering, University of Rome Tor Vergata,

Via del Politecnico 1, Roma, 00133, Italy

${ }^{2}$ Center for Advanced Biomaterials for Health Care, Istituto Italiano di Tecnologia,

Largo Barsanti e Matteucci 53, 80125 Naples, Italy

${ }^{3}$ School of Physics, National University of Colombia, Medellín Campus, A. A. 3840 Medellín, Colombia

${ }^{4}$ Cambridge Graphene Centre, Department of Engineering, University of Cambridge, 9 J J Thomson Avenue, Cambridge CB3 OFA, United Kingdom

* Correspondence to falconi@eln.uniroma2.it 


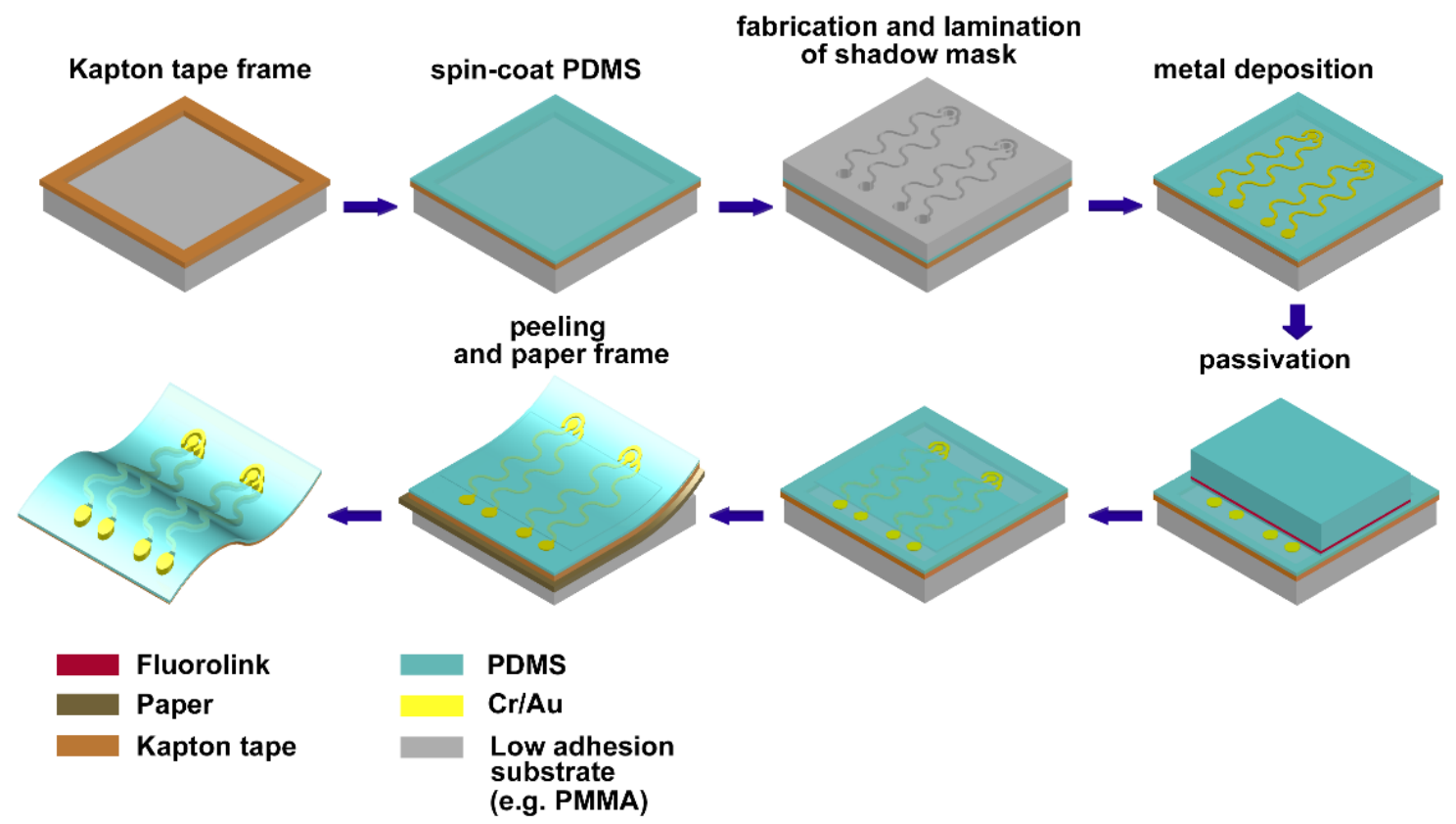

Figure S1. Complete process for fabricating double-framed contaminant-free thin PDMS devices.

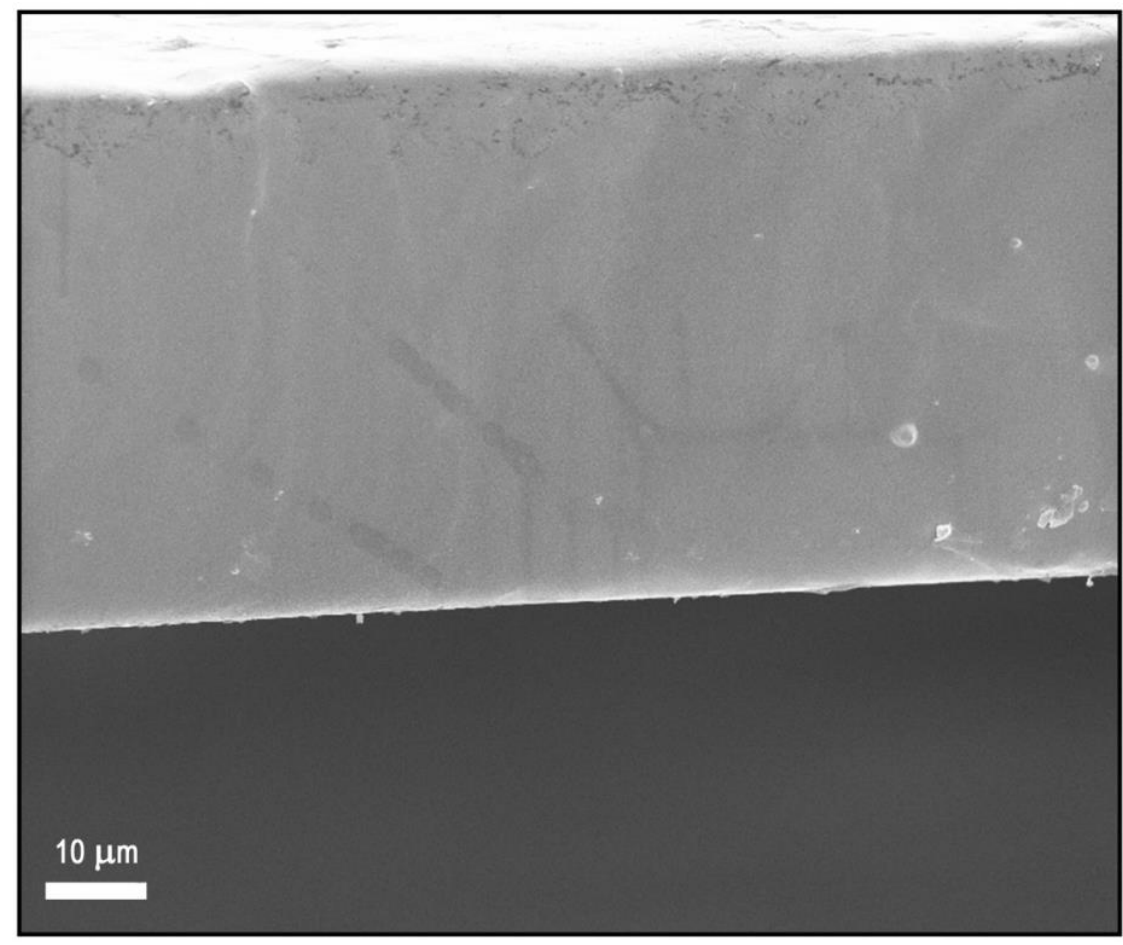

Figure S2. SEM cross section image of a $50 \mu \mathrm{m}$ PDMS device. 

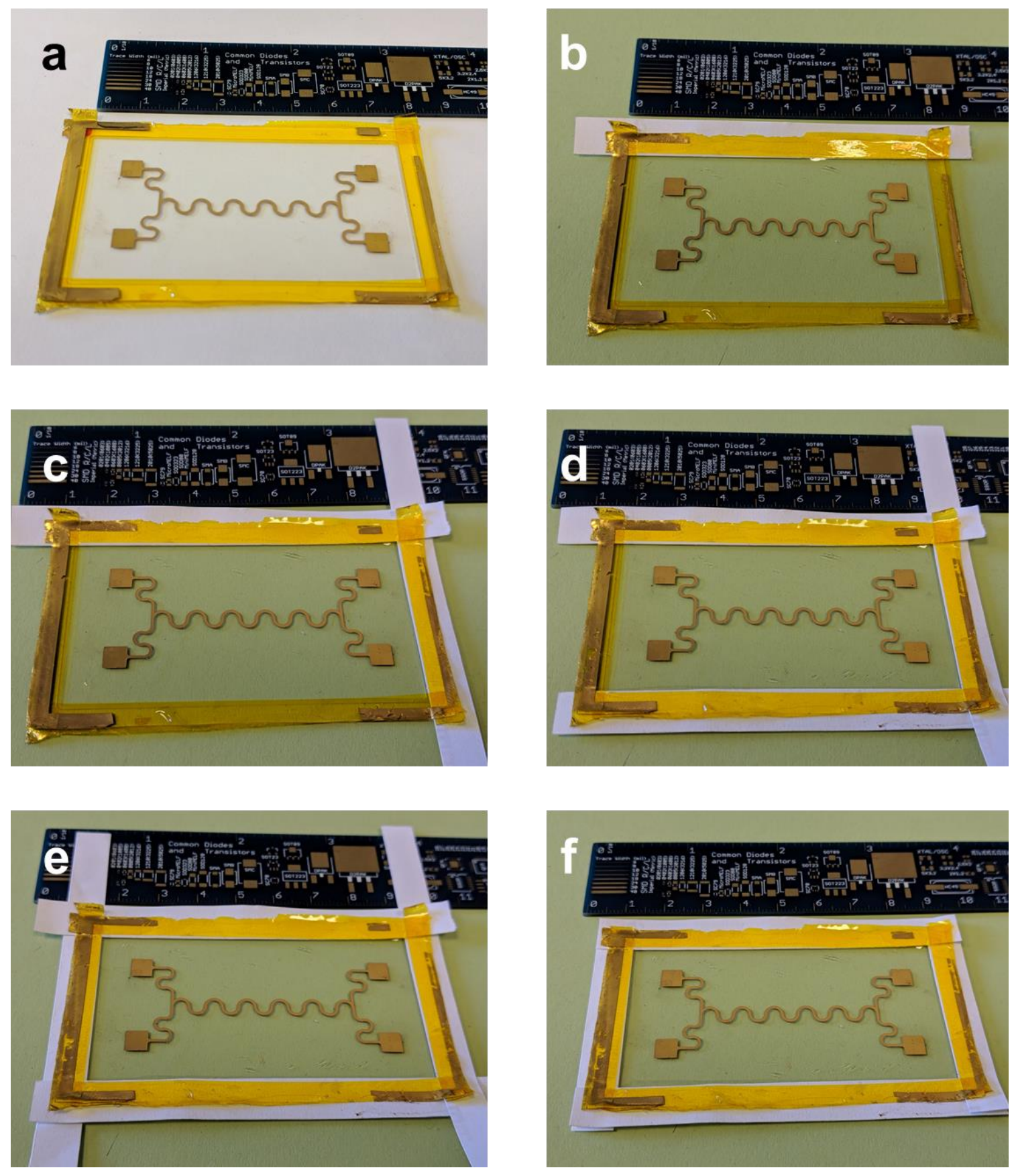

Figure S3. Side-by-side adhesive neutralization by auxiliary (non-adhesive) paper

frame. (a) PDMS device with a gold resistor on the PMMA substrate with Kapton frame.

(b-e) Neutralization of the Kapton adhesive by sequential attachment of paper to each side of the Kapton frame. (f) Removal of the excess paper, resulting in the double-framed device which can be easily removed from the low-adhesion PMMA substrate and, for easy handling, can be made somewhat more rigid by proper paper selection. 


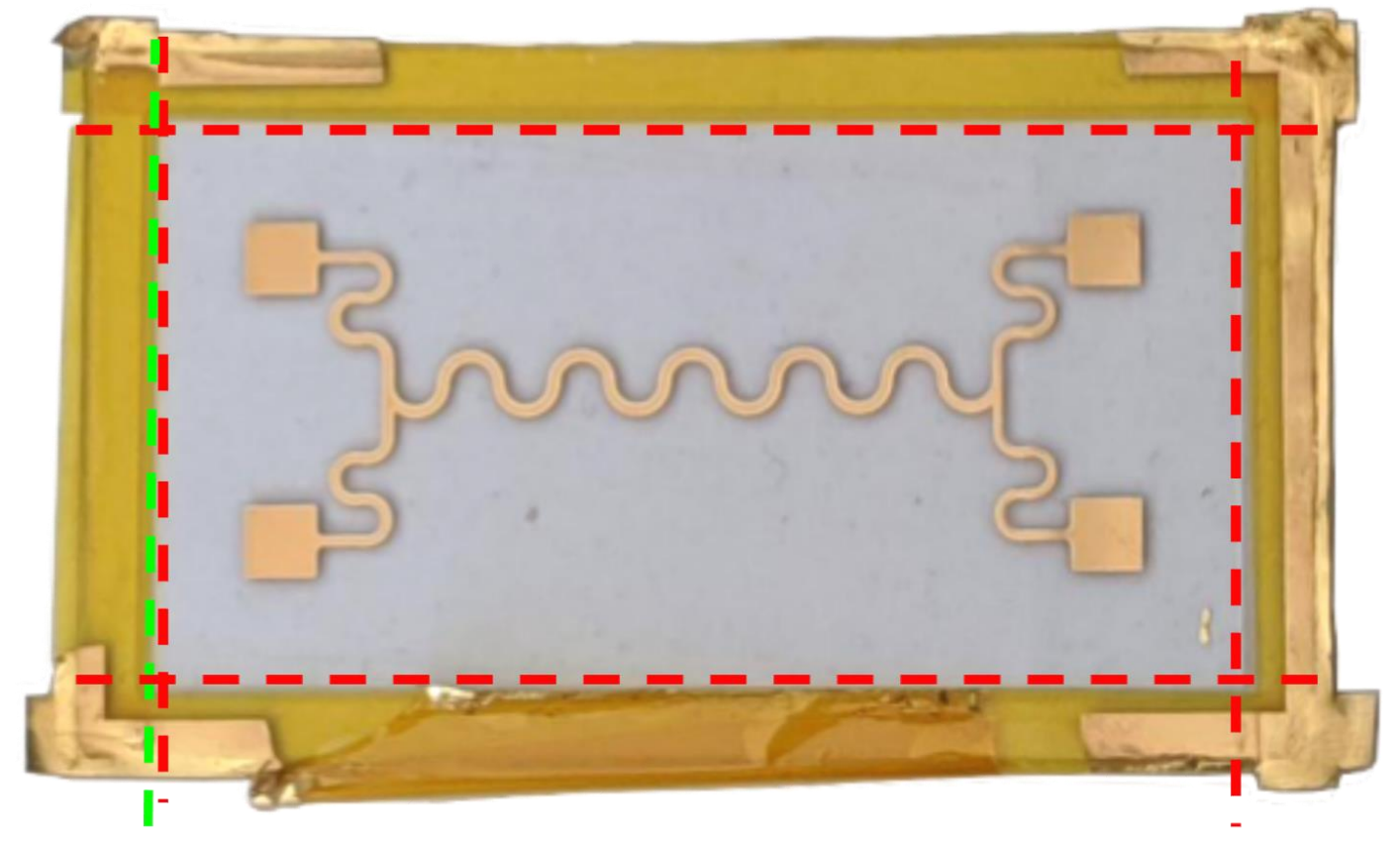

Figure S4. Optical image showing how marking lines micromilled on the carrier can reduce the Kapton tapes misalignment to very low angles. The Kapton tapes sides are almost perfectly parallel to the (ideally parallel and orthogonal) red dashed lines, except the left tape which has a misalignment around $0.5^{\circ}$.
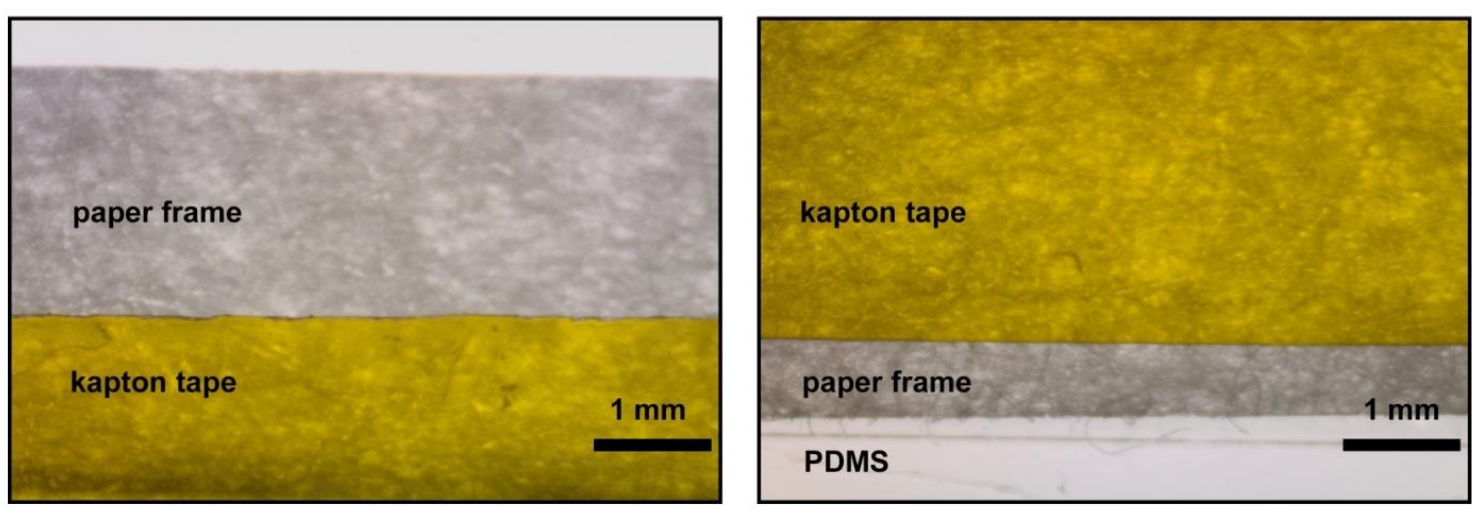

Figure S5. Optical microscope image of the Kapton-paper double frame (left) and of the complete PDMS-kapton-paper structure (right). 


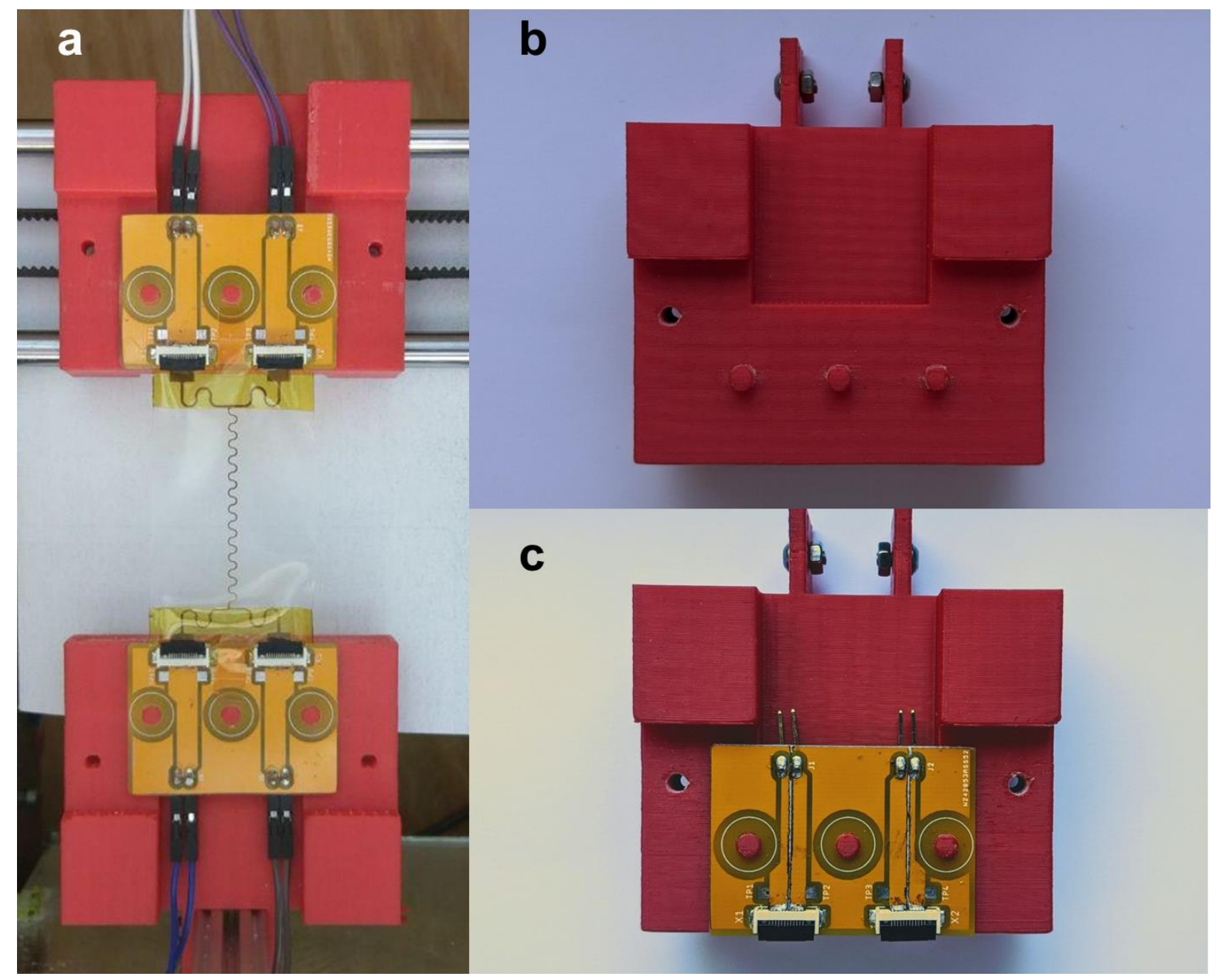

Figure S6. System for strain characterization. (a) System for applying controlled strain to the thin PDMS device by means of 3D printed holders (red-blocks), each having three small pillars, and PCBs (orange), each having three holes complementary to the three pillars; Kapton tapes have been attached on the bottom of the device in correspondence of the pads for locally increasing the device thickness, so that standard connectors can establish a good contact with the gold pads. (b-c) Zoom of the 3D printed holder before (b) and after (c) placement of the PCBs. 
a
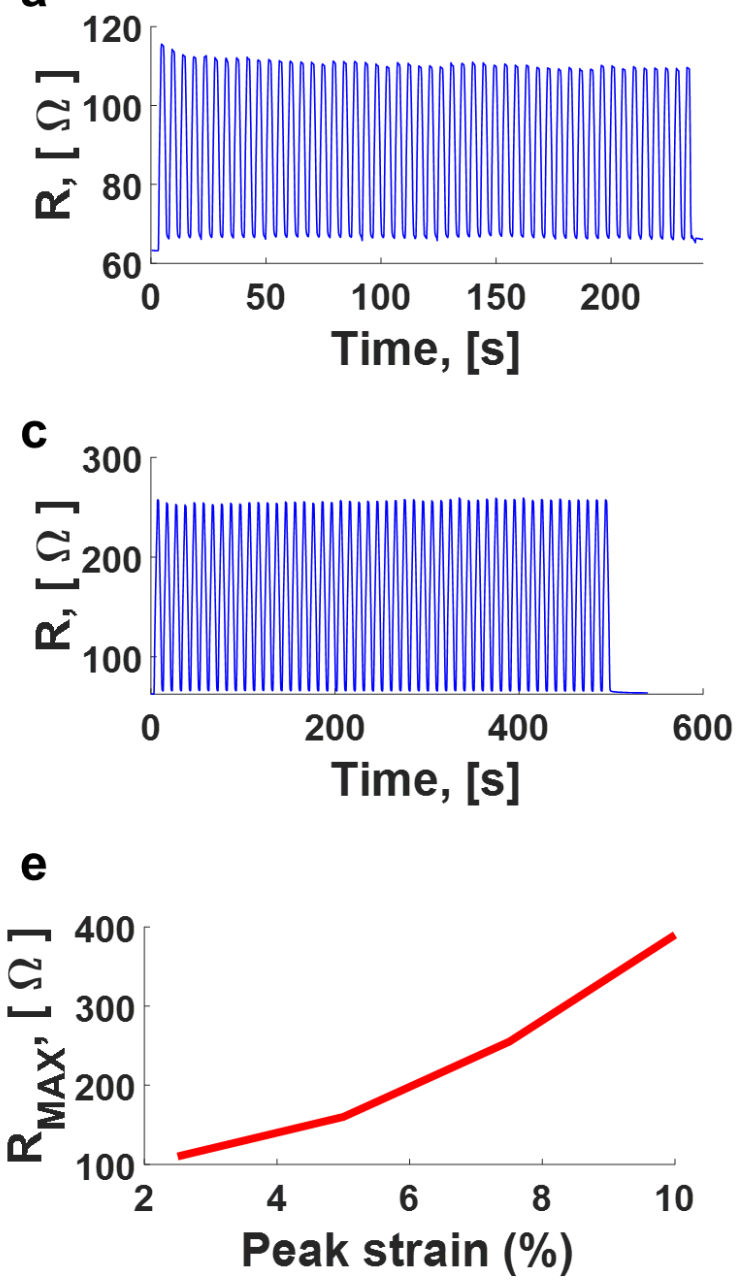

b

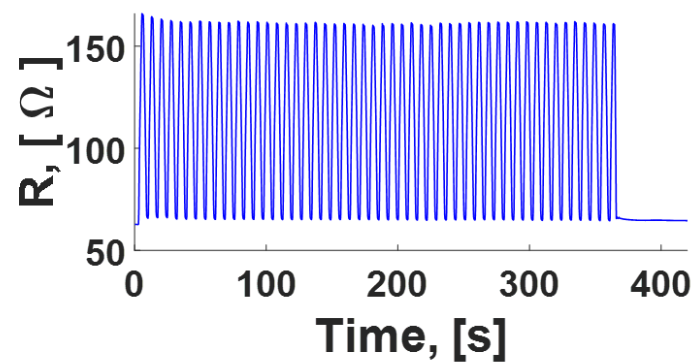

d

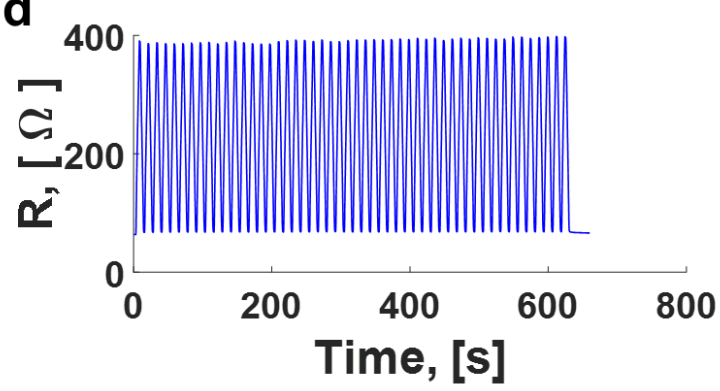

f

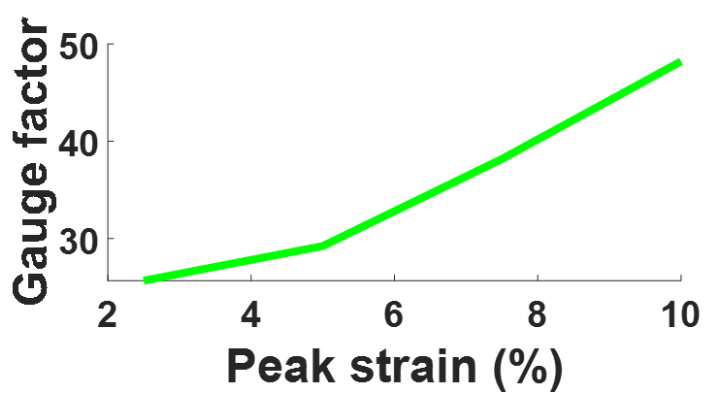

Figure S7. Strain characterization of thin gold resistors on PDMS. (a-d) 4-wires resistance measurement taken when applying 50 strain cycles with peak strains equal to $2.5 \%$ (a), $5 \%$ (b), $7.5 \%$ (c) and $10 \%$ (d), respectively. (e-f) Maximum resistance $\mathrm{R}_{\mathrm{MAX}}(\mathrm{e})$ and gauge factor (f) as a function of the peak strain for the 4-wires measurements (a-d). 
a

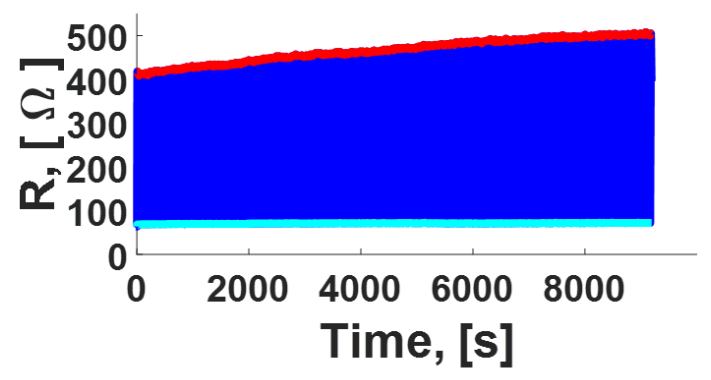

C

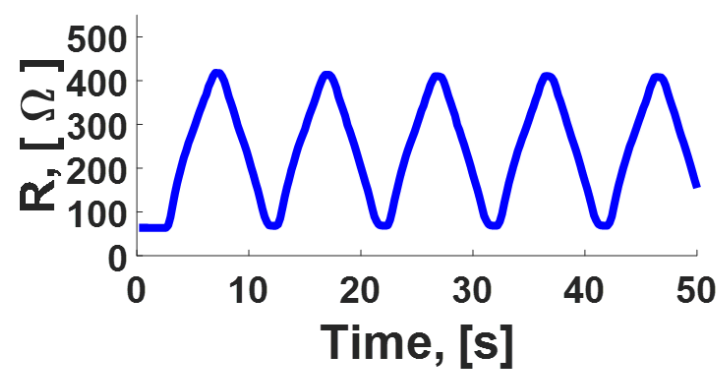

e

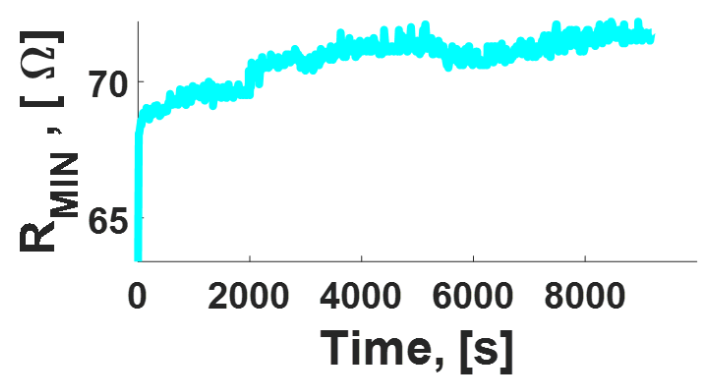

g

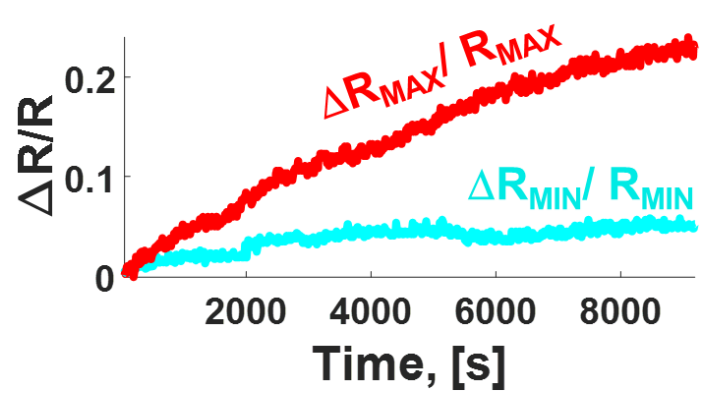

b

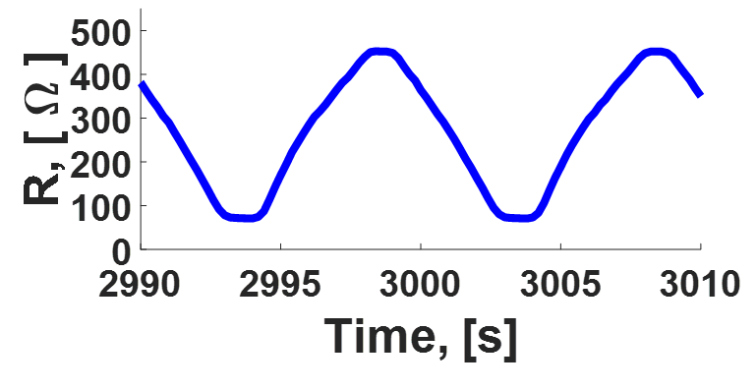

d

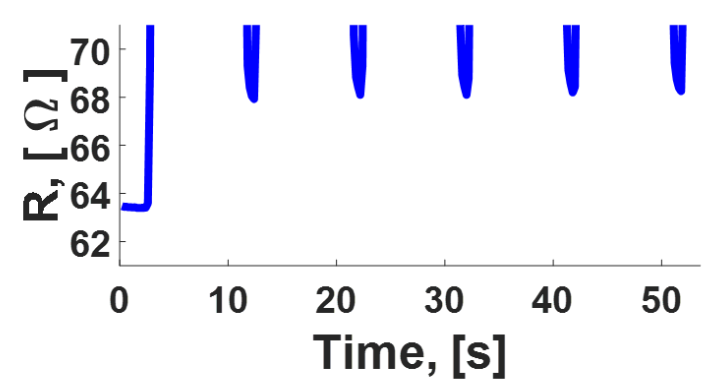

$\mathbf{f}$

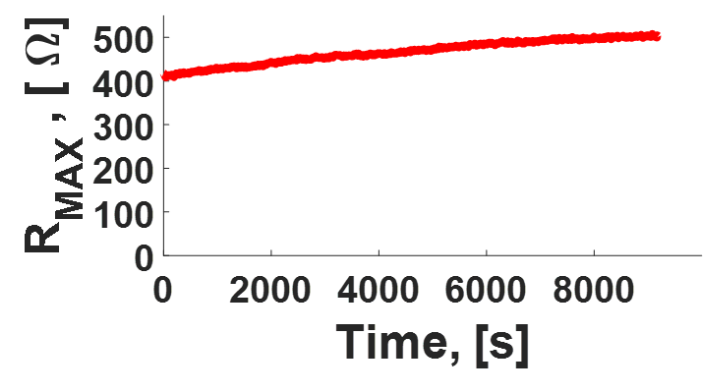

h

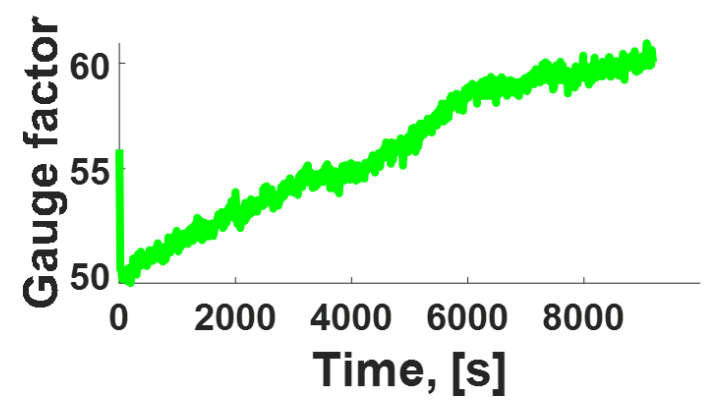

Figure S8. Analysis of 4-wires resistance measurements taken during strain cycles with $10 \%$ peak strain. (a) Time evolution of the resistance (blue), the maximum resistance (red) and the minimum resistance (cyan). (b) Zoom of the (almost periodic) time evolution of the resistance during two strain cycles (period equal to $10 \mathrm{~s}$ ). (c-d) Time evolution of the resistance 
in the initial 50 seconds (c) and zoom (d) which shows the initial step of the minimum resistance at the beginning of the strain cycles (the minimum resistance abruptly goes from about $63.5 \Omega$ to about $68 \Omega$ in the first cycle and then only slowly changes; the initial step may be attributed to residual strain in the resistor even when the device is under a nominally zero strain due to the PDMS inertia, i.e. viscoelasticity). (e) Time evolution of the minimum resistance; after the initial step (see (d)) at the beginning of the strain cycles, the minimum resistance slowly increases as a result of the gradual formation or growth of micro-cracks in the metals. (f) Time evolution of the maximum resistance which slowly increases as a result of the formation or growth of micro-cracks in the metals. (g) Time evolution of the relative variations of the minimum resistance (cyan) and of the maximum resistance (red), showing higher relative variations for the maximum resistance (for clarity and for a fair comparison, the measurements taken during the first $50 \mathrm{~s}$ have been ignored in order to exclude the initial step of the minimum resistance, see (d)). (h) Time evolution of an estimation for the gauge factor at the peak strain (computed by assuming that resistance at zero strain corresponds to the minimum resistance), showing that the gradual formation or growth of micro-cracks increases the gauge factor, as expected (since the relative variations of the maximum resistance are higher, see $(\mathrm{g})$, the relative increase of the gauge factor is approximately equal to the relative increase of the maximum resistance). 

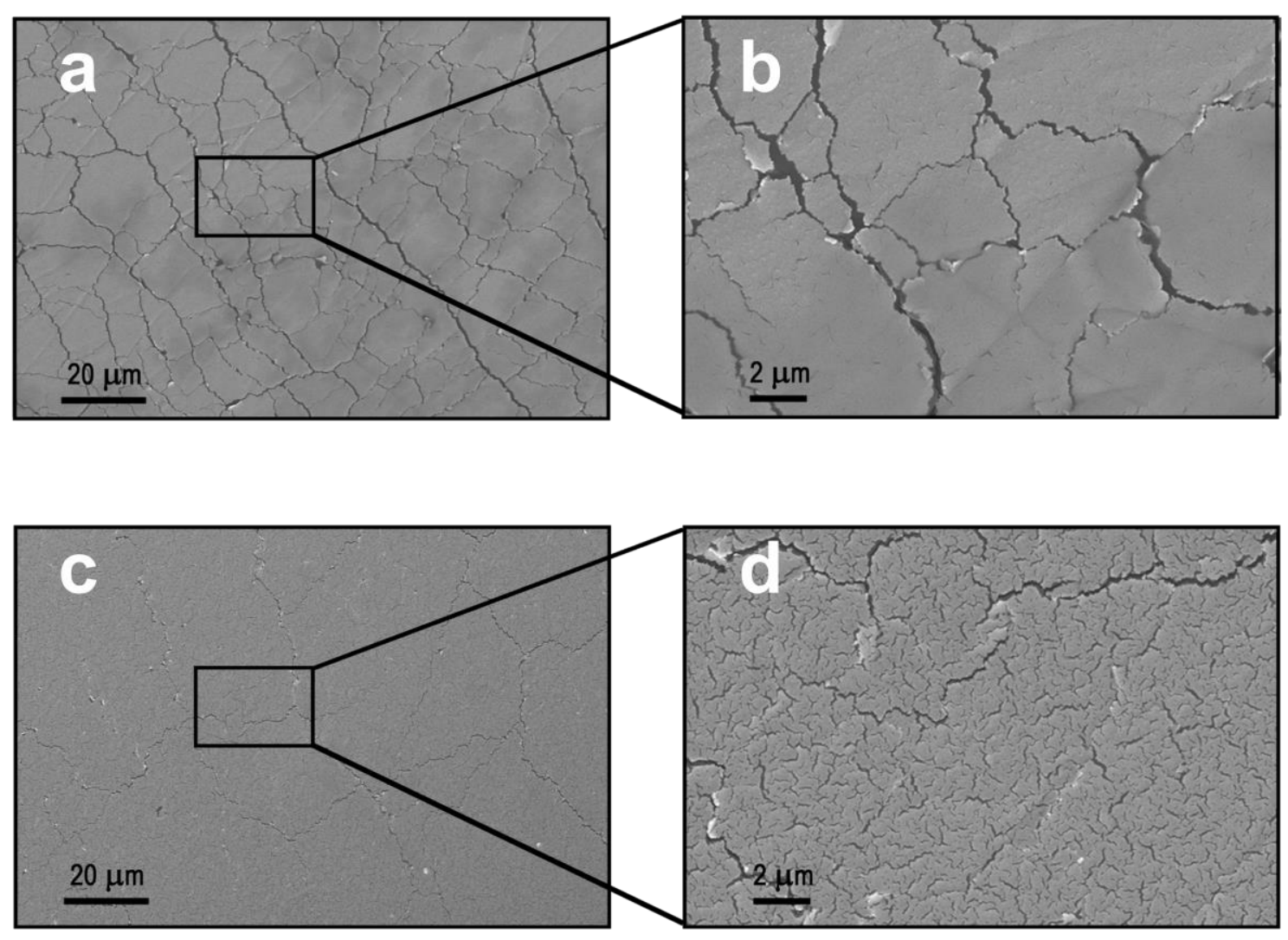

Figure S9. SEM images of metals after strain cycles. (a-b) SEM images of a serpentine device after 2000 strain cycles and 1\% strain increments until the electrical continuity of gold is temporarily lost. (c-d) SEM images of metal on a serpentine device after strain has been increased until PDMS breaks (at a 92.5\% strain). 


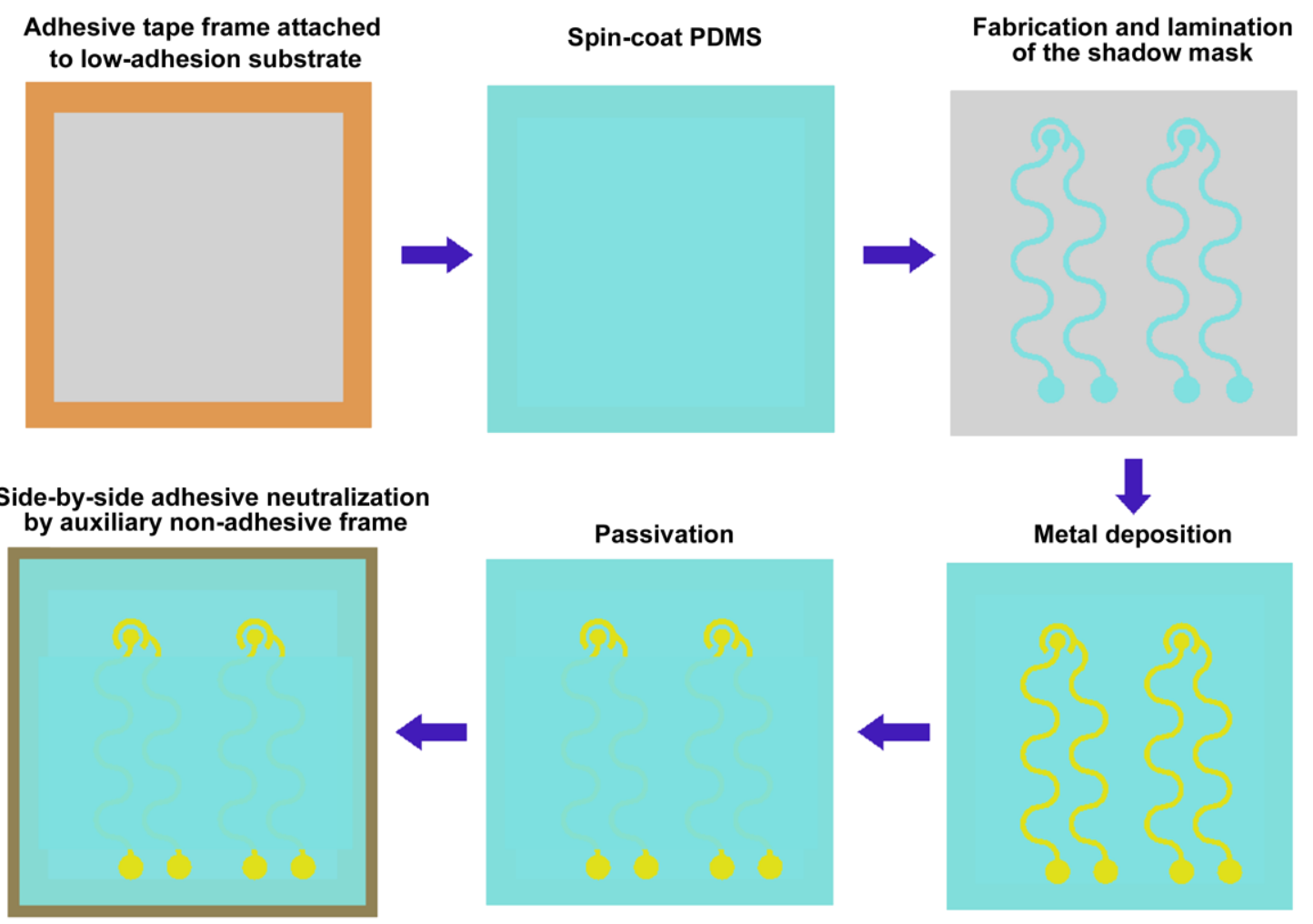

Figure S10. Schematic diagrams (top view) of the process for electrodes fabrication. All the metal traces, except the electrodes (for sensing) and the pads (for connecting to external instrumentation) are passivated. 
Table S1. Bioimpedance measurements taken with the 10 electrodes of a single epidermal device before (left column) and after (right column) treatment with a commercial moisturizing lotion.
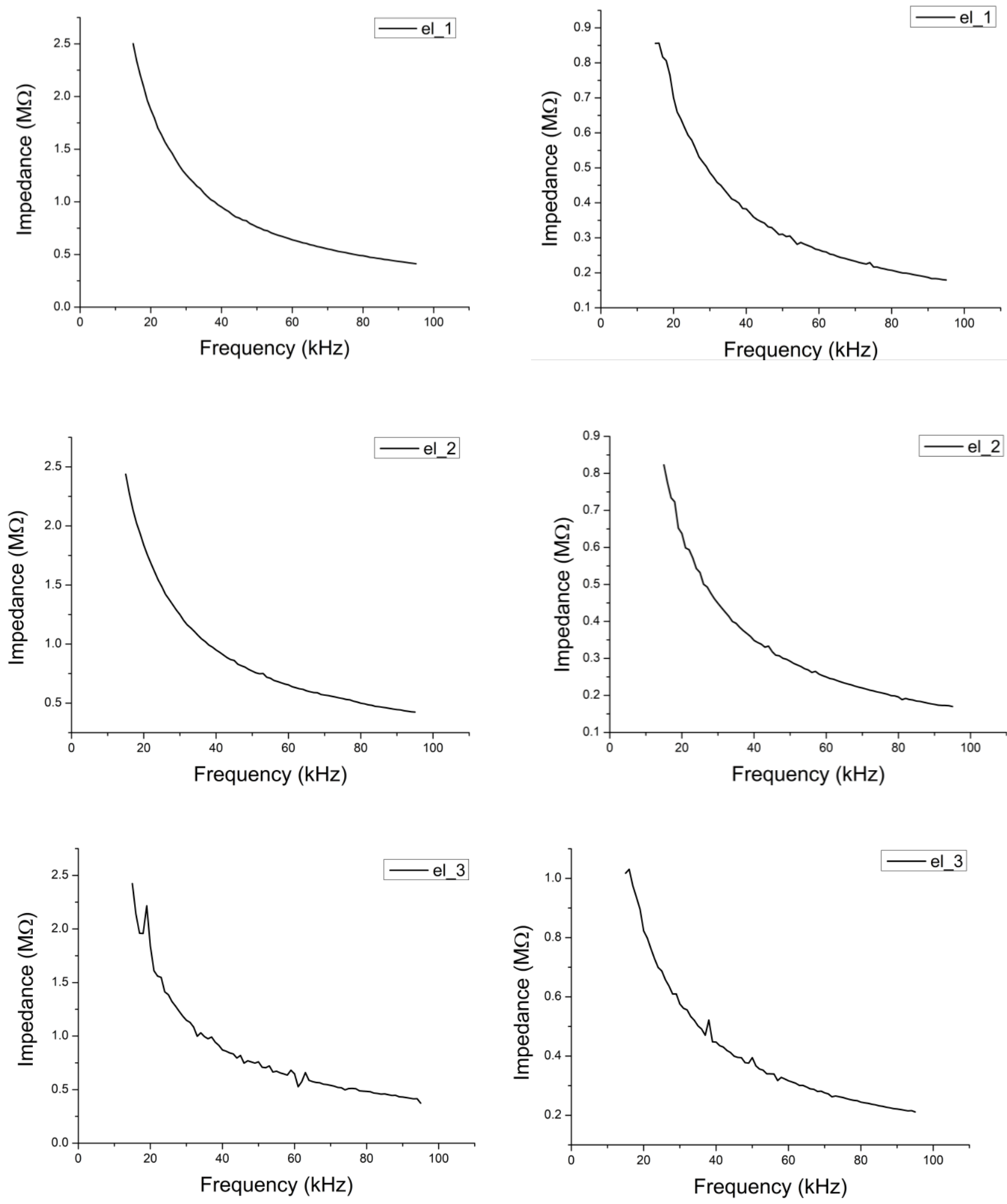

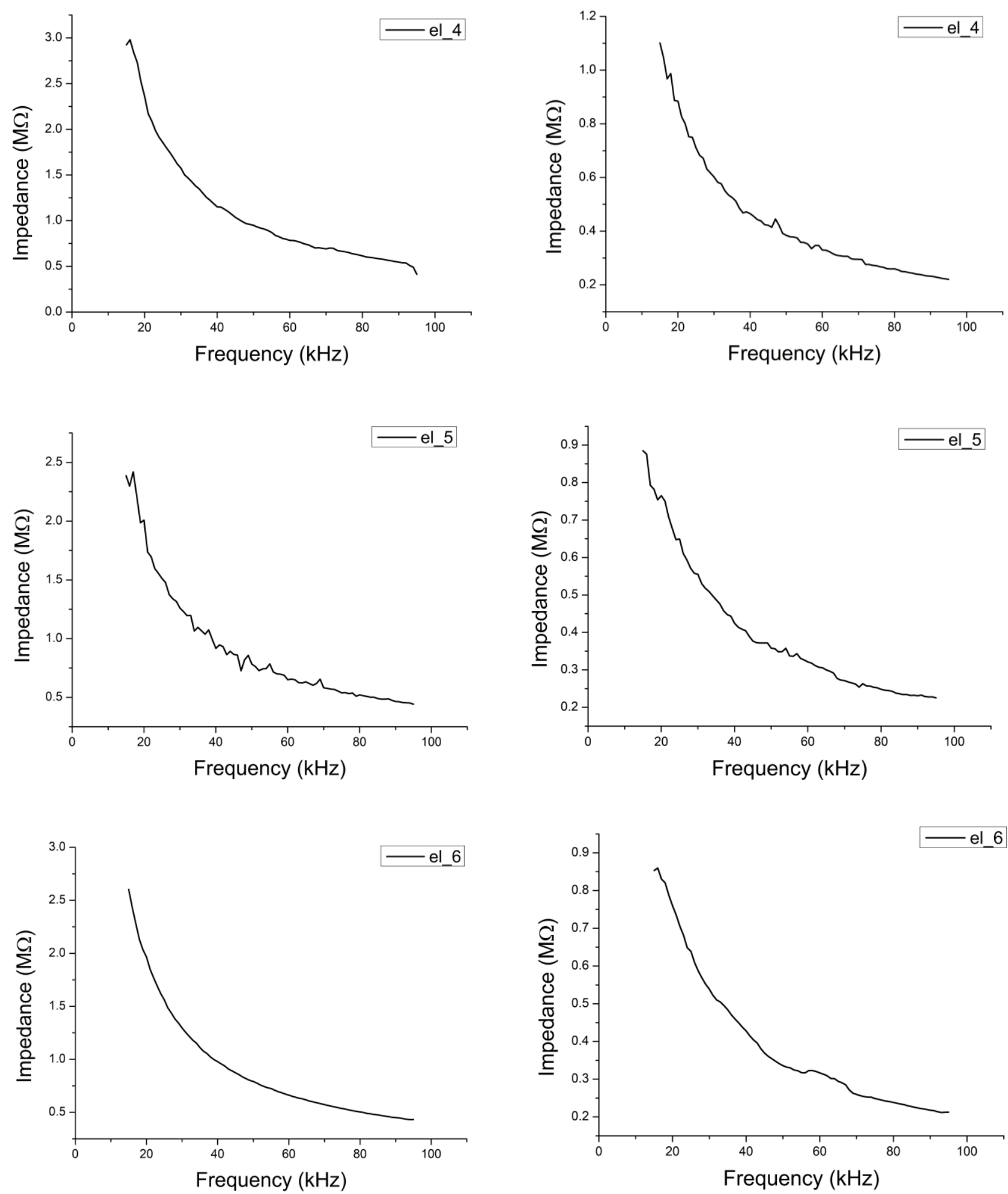

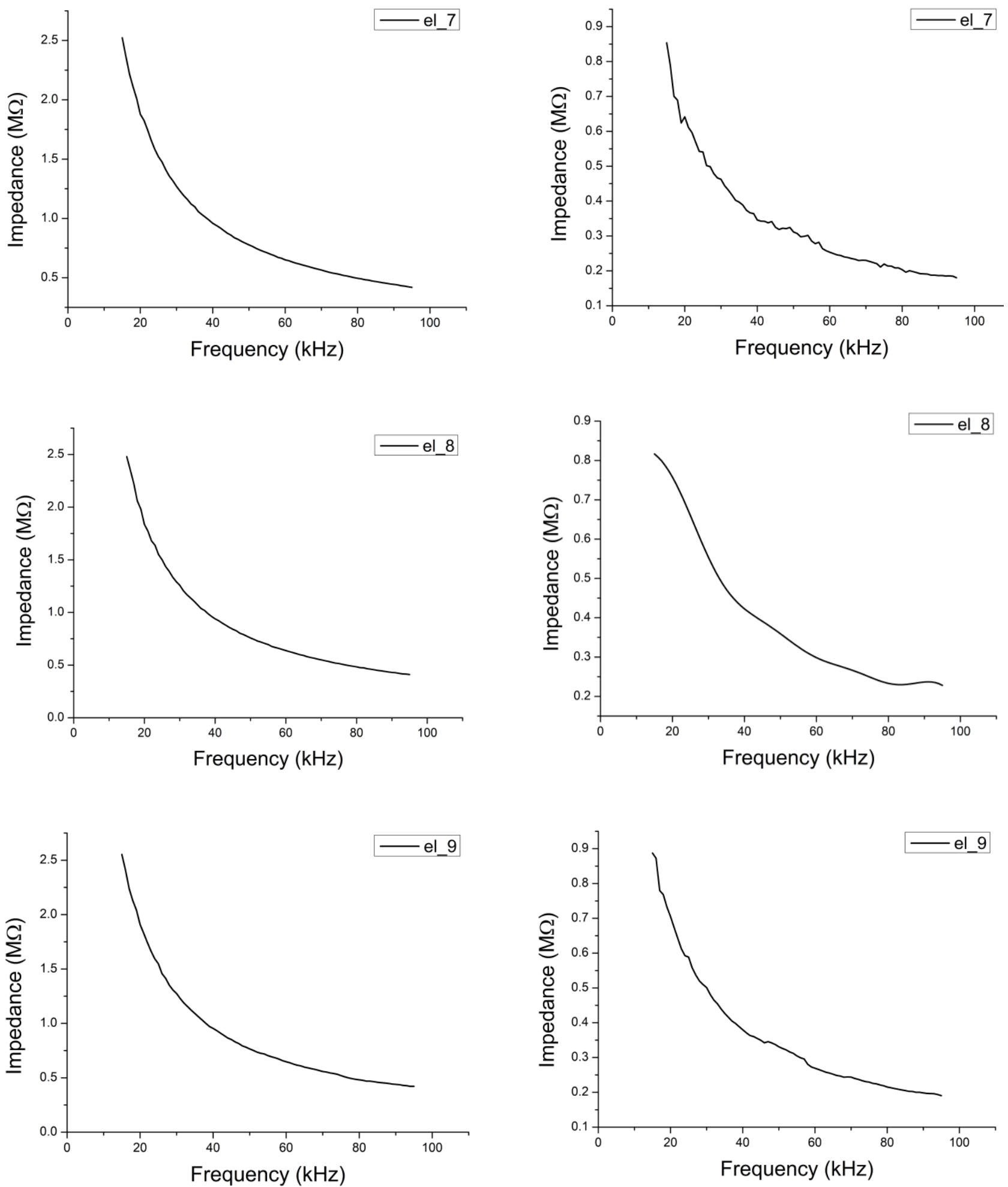

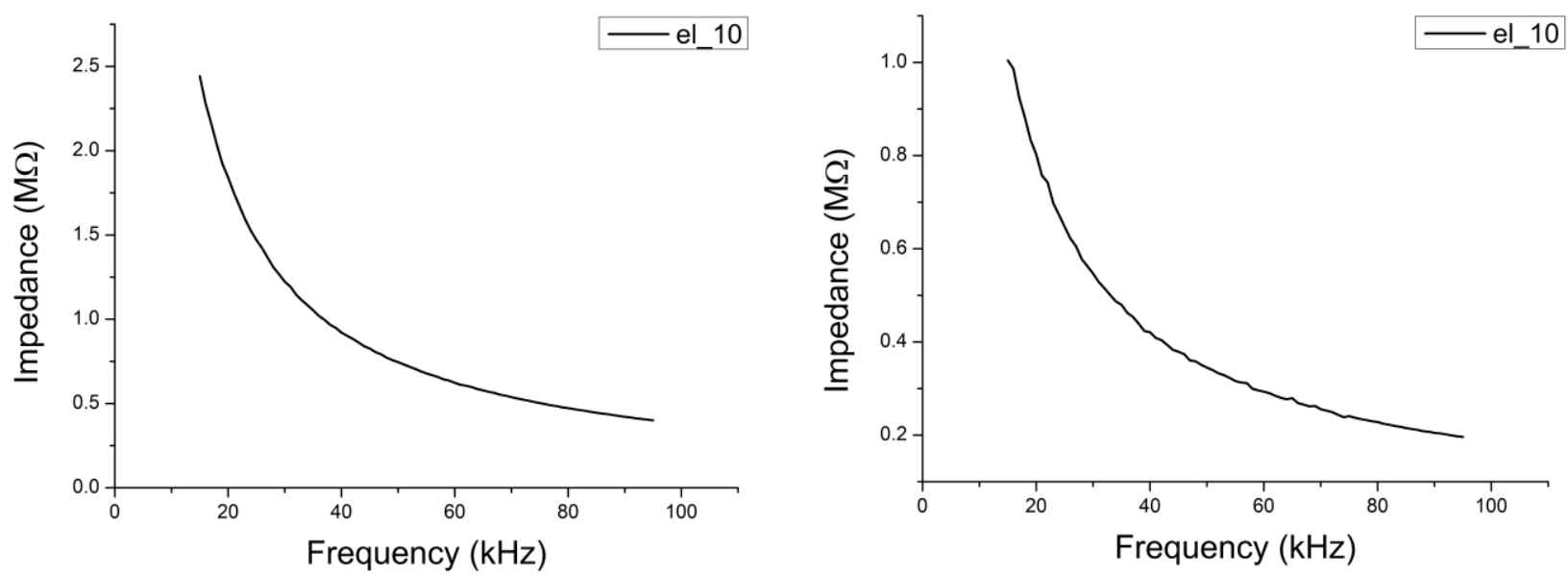